\title{
COMPLEX INTERPOLATION OF NORMED AND QUASINORMED SPACES IN SEVERAL DIMENSIONS. I
}

\author{
ZBIGNIEW SLODKOWSKI
}

\begin{abstract}
A variety of complex interpolation methods for families of normed or quasi-normed spaces, parametrized by points of domains in complex homogeneous spaces, parametrized by points of domains in complex homogeneous spaces, is developed. Results on existence, continuity, uniqueness, reiteration and duality for interpolation are proved, as well as on interpolation of operators. A minimum principle for plurisubharmonic functions is obtained and used as a tool for the duality theorem.
\end{abstract}

Introduction. The classical complex interpolation method for two spaces, due to Calderón, was substantially generalized by Coifman, Cwikel, Rochberg, Sagher and Weiss $[\mathbf{1}, \mathbf{2}]$. In their method, for a prescribed family of Banach spaces $X_{\zeta}$, parametrized by boundary points of the unit disc $D$, a family of intermediate Banach spaces, parametrized by points of $D$, is constructed.

The purpose of this paper is to develop a similar construction in the situation when $D$ is a domain in $\mathbf{C}^{k}, k>1$. In fact, our construction works when $D$ is a domain in a complex homogeneous space $M=K / H$. We obtain a variety of interpolation methods, each of them controlled by some invariant class $Q$ of functions on $M$.

All the basic results of Coifman et al. [1, 2], concerning existence, continuity, reiteration property and interpolation of operator norm, extend to all the methods we consider (cf. Theorem 2.3 and Corollaries $2.10,4.5$ below), but duality does not usually hold for an individual method. It turns out (cf. Theorem 6.1) that if the spaces $X_{\varsigma}, z \in D$, are constructed by the interpolation method corresponding to a class $Q$, then the dual spaces $X_{z}^{*}, z \in D$, form an interpolation family corresponding to the class of functions, denoted $Q^{d}$, which is dual, in a certain sense, to $Q$ (cf. $\S 1$ ). Still, self-dual methods do exist, in particular those corresponding to $Q=$ the class of all subharmonic functions on $M$ (cf. $\S \S 7.2(\mathrm{~b}), 8.2(\mathrm{c})$ ).

As for more technical aspects of this paper, we assume that all the spaces $X_{z}$ are finite-dimensional, $X_{z} \simeq \mathbf{C}^{n}$, and only the norms vary. (In fact, main results, except for the duality theorem, are proved for quasi-norms as well.) We also assume that the boundary spaces $X_{\varsigma}, \varsigma \in \partial D$, vary continuously. While these assumptions are clearly restrictive, it seems that the main analytic and geometric features of the

Received by the editors April 1, 1987.

1980 Mathematics Subject Classification (1985 Revision). Primary 32F05, 32F10, 32M10.

Key words and phrases. Interpolation spaces, subinterpolation and superinterpolation families, plurisubharmonic functions, subharmonic functions, pseudoconvex classes, Dirichlet problem, complex homogeneous spaces, strictly pseudoconvex domains.

The contents of this paper were presented at the International Conference on Harmonic Measure, Toledo (Ohio), July 1986. 
problem are already present here, and a generalization to the infinite-dimensional case should be now easier. The author intends to address this in a subsequent paper.

Although our arguments make considerable use of the complex structure, it seems still that the framework of pseudoconvex classes of functions might be useful for studying real interpolation methods as well.

In $\S 1$ we explain the motivation of our construction and review the notion of a pseudoconvex class of functions; $\mathrm{cf}$. $[\mathbf{1 8}, \mathbf{1 9}, \mathbf{2 0}]$ on which this construction is based. Specific examples of interpolation methods are discussed in $\S \S 7,8$ and the reader might want to have a look at these first. The general results are obtained in $\S \S 2-4$ and 6 . $\S 5$, which can be read independently, contains a minimum principle for plurisubharmonic functions, which generalizes a result due to Kiselman [5].

1. Pseudoconvex classes of functions. If $D$ is a set with a boundary $\partial D$, and $\left\{\mathbf{C}^{n}, p_{\zeta}\right\}, \zeta \in \partial D$, are prescribed normed spaces, the interpolation problem reduces to defining a family of intermediate norms $\tilde{p}_{z}: \mathbf{C}^{n} \rightarrow R, z \in D$. Geometrically, a family of circled, convex, compact neighborhoods of zero, $B(\varsigma)=\{w \in$ $\left.\mathbf{C}^{n}: p_{\varsigma}(w) \leq 1\right\}$, is given, and we have to construct an extension $z \rightarrow W(z): \bar{D} \rightarrow$ $2^{\mathrm{C}^{n}}$. Once this is done, $\tilde{p}_{\boldsymbol{z}}$ is defined as the Minkowski norm, or quasi-norm, determined by $W(z)$.

In our first method of construction $W(\cdot)$, it was defined as the largest (with respect to inclusion) upper semicontinuous set-valued function (shortly: multifunction) $z \rightarrow K(z): \bar{D} \rightarrow 2^{\mathbf{C}^{n}}$, which is analytic in $D$ in the sense of $[\mathbf{1 5}$, Definition 0.2 ], and such that $K(\varsigma) \subset B(\varsigma), \varsigma \in \partial D$. The resulting interpolation method has most of the desired properties but the duality fails: the dual norming bodies $W(z)^{0}$ do not usually form an interpolation family of the above type (i.e. $z \rightarrow W(z)^{0}$ is not an analytic multifunction).

The search for other interpolation methods aimed first at identifying the dual balls $W(z)^{0}$ as a certain interpolation family, and then at finding some interpolation methods which have the self-duality property. Recall that a multifunction $z \rightarrow K(z): D \rightarrow 2^{\mathbf{C}^{n}}$ is analytic if and only if its graph $\operatorname{gr}(K)=\{(z, w): z \in G$, $w \in K(z)\}$ has the local maximum property with respect to the class of $(k-1)$ plurisubharmonic functions on $D \times \mathbf{C}^{n}$, where $k=\operatorname{dim}_{\mathbf{C}} D$; cf. [15, Definition 0.2 ; 13, Theorem 5.1]. It was further proved (cf. §6) that the graph of the correspondence $z \rightarrow W(z)^{0}: D \rightarrow 2^{\mathbf{C}^{n}}$ had the local maximum property relative to the class of all plurisubharmonic functions on $D \times \mathbf{C}^{n}$, and was the largest one with this property.

These observations suggest to consider a class of functions, say $P$, on $D \times \mathbf{C}^{n}$, and define $P$-interpolation family $z \rightarrow W(z): \bar{D} \rightarrow 2^{\mathrm{C}^{n}}$ as the largest multifunction with given boundary sections $B(\varsigma)$ and such that functions from $P$ have local maximum property on $\operatorname{gr}(K \mid D)$. We mostly use special classes $P=P_{Q}^{\text {co }}$ or $P_{Q}^{\text {psh }}$ (cf. Definition 1.1) which are determined, in a functorial way, by a class $Q$ of $D$. We consider classes $Q$ and $P$ which satisfy conditions (1.1) (1.13). Classes $Q$ are defined on subsets of a space $M$, and classes $P$ are defined on subsets of $\tilde{M}=M \times \mathbf{C}^{n}$. The set of all functions of class $Q$ defined on $U$ is denoted by $Q(U)$. Axioms (1.1) (1.13) are formulated for $Q$; a formulation for $P$ is given only when it is essentially 
different.

(1.0) the structure of a complex homogeneous space $K / H$, where $K, H$ are real Lie groups;

(i) $Q(U) \subset \operatorname{usc}(U)=$ the class of all upper semicontinuous functions on $U$, if $U \subset M$; (ii) $u \in Q(U)$ if and only if $u \in \operatorname{usc}(U)$ and $u \in Q(U \backslash \Gamma) ; u \in P(V), V \subset \tilde{M}$, if and only if $u \in \operatorname{usc}(V)$ and $u$ is of class $P$ on $V \backslash\left(\Gamma \times \mathbf{C}^{n}\right)$;

$$
\begin{aligned}
& \text { if } U \subset V \text { and } u \in Q(V) \text {, then } u \mid U \in Q(U) \text {; } \\
& \text { if } u_{n} \in Q(U), u_{n}(x) \searrow u(x), x \in U \text {, then } u \in Q(U) ;
\end{aligned}
$$

the usc regularization of the supremum of a locally uniformly bounded (from the above) subfamily of $Q(U)$ belongs to $Q(U)$;

if $u \in Q(U), C \in R$, then $(u+C) \in Q(U)$;

$Q(U)$ contains bounded functions (follows from (1.10));

$Q$ is a sheaf; that is, if $u \in \operatorname{usc}(U), U=\bigcup_{t} U_{t}$ and $u \mid U_{t} \in Q\left(U_{t}\right)$ for each $t$, then $u \in Q(U)$;

if $u \in Q\left(U_{1}\right)$ and $v$ is plurisubharmonic on $U_{2} \subset M \backslash \Gamma$, then $(u+$ $v) \in Q\left(U_{1} \cap U_{2}\right)$; if $u(z, w)$ is of class $P$ on $V_{1} \subset M \times \mathbf{C}^{n}$, and $v(z, w)$ is plurisubharmonic on $V_{2} \subset(M \backslash \Gamma) \times \mathbf{C}^{n}$, and for each $z$, the slice function $w \rightarrow v(z, w)$ is convex, then $(u+v) \in P\left(V_{1} \cap V_{2}\right)$;

(i) for $g \in H$, let $t_{g}$ be the biholomorphic map of $H / K$ onto itself, defined by $t_{g}(h K)=(g h) K$; class $Q$ is invariant with respect to maps $t_{g}, g \in K$; class $P$ is invariant with respect to maps $(z, w) \rightarrow\left(t_{g} z, w\right)$; (ii) class $P$ is invariant with respect to vertical translations $(z, w) \rightarrow\left(z, w+w_{0}\right), w_{0} \in \mathbf{C}^{n}$; (iii) the same as (ii) for homotheties $(z, w) \rightarrow(z, t w), t>0$; (iv) the same for $(z, w) \rightarrow(z, \alpha w), \alpha \in \mathbf{C}, \alpha \neq 0$; (v) class $P$ is invariant with respect to maps $(z, w) \rightarrow(z, f(z) w)$, where $f(z)$ is a nowhere vanishing holomorphic function;

constant functions belong to $Q(U)$;

if $K \subset M \backslash \Gamma$ is compact and $u \in Q($ nbhd $K)$, then $\max u \mid K \leq$ $\max u \mid \partial K$; if $u(z, w)$ is of class $P$ in a neighborhood of $K \times\{0\}$ in $M \times \mathbf{C}^{n}$, then $\max _{z \in K} u(z, 0) \leq \max _{z \in \partial K} u(z, 0)$;

if $u \in Q(U), r>0$, then $r u \in Q(U)$;

if $v$ is a convex function on $\mathbf{C}^{n}$, then $(z, w) \rightarrow v(w)$ is a function of class $P$. 
Conditions (1.1)-(1.9) imply that $Q, P$ are pseudoconvex classes of functions; see $[18, \S 1]$ for more detailed discussion of the above axioms, and $[18, \S 2 ; 19]$ for examples of pseudoconvex classes. In particular, the classes of all $q$-plurisubharmonic functions on $M$ or $\tilde{M}$, denoted further by $P_{q}$, are examples of $Q$ or $P$ above, cf. $[12, \S 1]$.

DEFINITION 1.1 [20, Definition 4.1]. If $Q$ is a class of functions on $M$, we define class $P_{Q}^{\text {psh }}$ and $P_{Q}^{\text {co }}$ on $\widetilde{M}=M \times \mathbf{C}^{n}$. A usc function $u(z, w)$ belongs to $P_{Q}^{\mathrm{psh}}$ if, for every analytic map $z \rightarrow F(z)$, the composition $z \rightarrow u(z, F(z))$ is of class $Q$ and slice functions $w \rightarrow u(z, w)$ are plurisubharmonic. If, in addition, slice functions are convex, $u$ belongs to $P_{Q}^{\text {co }}$.

By [20, Propositions 4.2-4.4], if $Q$ satisfies (1.1)-(1.12), then so do $P_{Q}^{\text {co }}$ and $P_{Q}^{\mathrm{psh}}$.

Recall after [18, Definition 1.11] that $u \in Q^{d}\left(U_{1}\right)$, if $u+v$ has local maximum property on $U_{1} \cap U_{2}$, whenever $v \in Q\left(U_{2}\right)$. See $[18, \S 2]$ for basic properties of the dual class $Q^{d}$.

If $Q$ satisfies (1.0)-(1.12), then

$$
\begin{array}{r}
Q+Q^{d} \subset P_{k-1}, \quad \text { where } \operatorname{dim}_{\mathbf{C}}(M \backslash \Gamma)=k ; \\
Q^{d d}=Q ; \\
P_{0} \subset Q \subset P_{k-1} ; \\
\left(P_{q}\right)^{d}=P_{k-q-1}, \quad \text { if } q=0,1, \ldots, k-1 .
\end{array}
$$

Inclusion (1.14) follows from the definition of $Q^{d}$, condition (1.8) and [12, Propositions 1.1(i), 1.2(v)]; (1.16) follows from (1.10) and (1.8); (1.15) was proved in [20, $\S 5]$ and (1.17) is a consequence of [12, Proposition 1.1(i) and Theorem 5.1].

DEFINITION 1.2 [18, DEFINITION 1.3]. We define $A Q$, the additive kernel of $Q$, as the class of all functions $u \in \operatorname{usc}(U), U \subset M$, such that $u+Q \subset Q$.

Axiom 1.8 means that $A Q \supset P_{0}$. The next fact generalizes [15, Proposition 3.4].

PROPOSITION 1.3. Let $M, \Gamma, Q$ satisfy (1.10) - (1.12), and $z \rightarrow K(z): G \rightarrow$ $2_{2} \mathrm{C}^{n}, G \subset M$ open, be a usc, compact-valued function. Assume that $\operatorname{gr}(K)$ has local maximum property with respect to functions of class $P_{Q}^{\mathrm{psh}}$. Let $u(z, w, \xi)$ be a function of class $P_{Q}^{\text {psh }}$ on a neighborhood of $\operatorname{gr}(K) \times \mathbf{C}^{s}$ in $M \times \mathbf{C}^{n+s}$, and $v(z, \xi)=\max \{u(z, w, \xi): w \in K(z)\}, z \in G, \xi \in \mathbf{C}^{s}$. Then $v \in P_{k-1}\left(G \times \mathbf{C}^{s}\right)$, where $k=\operatorname{dim}_{\mathbf{C}} M$.

(a) In general, $v \in P_{(A Q)^{d}}^{\mathrm{psh}}\left(G \times \mathbf{C}^{s}\right)$. In particular, if $Q^{d}$ is closed with respect to addition, then $v \in P_{Q}^{\mathrm{psh}}\left(G \times \mathbf{C}^{s}\right)$.

Proof. Assertion. If $R$ is a pseudoconvex class of functions on $M$ (i.e. (1.1)(1.12) hold), such that $R+Q \subset Q$, then $v \in P_{R^{d}}^{\mathrm{psh}}\left(G \times \mathbf{C}^{s}\right)$.

The assertion implies the proposition. If we let $R=P_{0}$, then by (1.8), $R+Q \subset Q$, and $R^{d}=P_{0}^{d}=P_{k-1}$, by (1.17). Hence $v \in P_{P_{k-1}}^{\mathrm{psh}}$. The latter class is equal to $P_{k-1}$ (on $M \times \mathbf{C}^{n}$ ) by [20, Proposition 4.7].

As for part (a), if $R=A Q$, then $R+Q \subset Q$ by Definition 1.2. If $Q^{d}$ is closed with respect to addition, then $Q^{d}+\left(Q^{d}+Q\right) \subset Q^{d}+Q$. Hence, $Q^{d}+\left(Q^{d}+Q\right)$ consists of functions with local maximum property, which means that $Q^{d}+Q \subset Q^{d d}$, by $[\mathbf{1 8}$, 
Definition 1.11]. By (1.15), $Q^{d}+Q \subset Q$, and so, if we let $R=Q^{d}$, the assertion implies that $v \in P_{R^{d}}^{\mathrm{psh}}=P_{Q}^{\mathrm{psh}}$, since $R^{d}=Q$ by $(1.15)$.

To prove the assertion, observe first that the upper semicontinuity of $v$ is obvious, and since the slice function $\xi \rightarrow v\left(z_{0}, \xi\right)$ is usc and equal to the supremum of functions $\left\{\xi \rightarrow u\left(z_{0}, w, \xi\right)\right\}_{w \in K\left(z_{0}\right)}$, it is plurisubharmonic. By Definition 1.1 and [18, Definition 1.11], we have to check that if $F: G_{1} \rightarrow \mathbf{C}^{s}, G_{1} \subset G \backslash \Gamma$, is a holomorphic map and $f \in R\left(G_{2}\right), G_{2} \subset M$, then $\varphi(z)=f(z)+v(z, F(z))$ has local maximum property on $G_{1} \cap G_{2}$.

To see this, let $\psi(z, w)=f(z)+u(z, w, F(z))$. Function $\psi$ is of class $P_{Q}^{\text {psh }}$ on a neighborhood of $\operatorname{gr}(K)$ in $G \times \mathbf{C}^{n}$. Indeed, it is plurisubharmonic on fibers $\{z\} \times \mathbf{C}^{n}$, and for every analytic map $F_{1}: G_{0} \rightarrow \mathbf{C}^{n}$, the composition $z \rightarrow$ $u\left(z, F_{1}(z), F(z)\right)$ belongs to $Q$, and since $R+Q \subset Q$, the sum $z \rightarrow \psi\left(z, F_{1}(z)\right)=$ $f(z)+u\left(z, F_{1}(z), F(z)\right)$ is of class $Q$. By assumptions, $\psi \mid \operatorname{gr}(K)$ has local maximum property. Seeing that $\varphi(z)=\max \{\psi(z, w): w \in K(z)\}$, we conclude that $\varphi$ has local maximum property on $G$ (cf. $[18,(1.11)])$. Q.E.D.

TERMINOLOGY AND NOTATION. By a norming body in $\mathbf{C}^{n}$ we mean a compact, circled and convex neighborhood of zero.

It is easy to check that a bounded star-shaped neighborhood of zero in $\mathbf{R}^{N}$ has a continuous Minkowski functional, if and only if it is strictly star-shaped in the sense that every ray $\{r x: r>0\}$ intersects its boundary at precisely one point. By a quasi-norming body in $\mathbf{C}^{n}$ we understand a circled, strictly star-shaped and compact neighborhood of zero. Consequently, all quasi-norms in this paper are assumed to be continuous and, whenever defined in $\mathbf{C}^{n}$, condition $p\left(e^{i \theta} z\right)=p(z)$ is assumed.

We collect for further reference the following folklore.

A quasi-norming body in $\mathbf{C}^{n}$ is polynomially convex, if and only if its interior is pseudoconvex, which holds if and only if the corresponding Minkowski functional is plurisubharmonic on $\mathbf{C}^{n}$.

If $W(z), z \in D$, are quasi-norming bodies, $p_{z}(w)$ are corresponding Minkowski functionals (and $D$ is locally compact), then $z \rightarrow$ $W(z): D \rightarrow 2^{\mathbf{C}^{n}}$ is an usc multifunction (in the usual sense), if and only if $(z, w) \rightarrow p_{z}(w)$ is an lsc (=lower semicontinuous) function.

In the same notation, the function $(z, w) \rightarrow p_{z}(w)$ is usc, if and only if $z \rightarrow W(z)$ is lsc in the following sense: for every $z_{0}$ and for every compact set $K \subset \operatorname{Int} W\left(z_{0}\right)$, the set $\{z \in D: K \subset$ Ind $W(z)\}$ is open. A version with lower semicontinuity of $W(\cdot)$ in the usual sense still holds for norming, but not for quasi-norming, bodies.

2. The Perron method for set-valued functions. In this section we prove existence and continuity of interpolation families (Theorem 2.3).

DEFINITION 2.1 (i). Let $P$ be a class of usc functions on $\widetilde{M}$, and $K \subset Y \subset \widetilde{M}$, where $K$ is compact and $Y, \widetilde{M}$ are locally compact. Recall after $[\mathbf{1 8}$, Definition 4.11] that the saturation of $K$ relative to $Y$ (and $P$ ), denoted by $\operatorname{Sat}_{Y}(K)$, is the union of all compact sets $Z \subset Y$, such that $Z \backslash K$ is a $P$-maximum set, i.e. functions of class $P$, restricted to $Z \backslash K$, have local maximum property in the sense of (1.11) above; cf. [18, Definition 4.1]. 
(ii) Let $P$ be a class of functions on $\widetilde{M}=M \times R^{N}$, satisfying conditions (1.1)(i), (1.11). Let $\zeta \rightarrow B(\varsigma): \partial G \rightarrow 2^{R^{N}}$ be an usc multifunction, where $B(\varsigma)$ are compact, star-shaped neighborhoods, of zero, and $G \subset M \backslash \Gamma$ is open and relatively compact in $M$. Since, by (1.11), $G \times\{0\}$ is a $P$-maximum set, the compact set $\bar{G} \times\{0\}$ is contained in $\operatorname{Sat}_{\bar{G} \times R^{N}}(\operatorname{gr}(B))$, and so the latter set is the graph of some multifunction $z \rightarrow W(g): \bar{G} \rightarrow 2^{R^{N}}$. We will call the multifunction $W(\cdot)$ the Perron envelope of boundary data $B(\cdot)$ (relative to $P$ ).

DEFINITION 2.2. Let $Q$ be a class of functions on $M$ and $G \subset M$ be relatively compact. We say that $G$ is $Q$-regular, if for every $\varsigma \in \partial G$ there is a "barrier" function $u^{\varsigma} \in P(G)$, such that $u^{\varsigma}(\varsigma)=0, u^{\varsigma} \mid \bar{G} \backslash\{\zeta\}<0$ and $\lim _{z \rightarrow \varsigma} u^{\varsigma}(z)=0$. (Function $u$ lies in $P(\bar{G})$ if there are functions $u_{n} \in P(\operatorname{nbhd} \bar{G})$, such that $u_{n}(x) \searrow$ $u(x), x \in \bar{G}$.)

THEOREM 2.3. Let $M, \Gamma, Q$ satisfy conditions (1.0)-(1.12). Let $\varsigma \rightarrow B(\varsigma): \partial G$ $\rightarrow 2^{\mathrm{C}^{n}}$ be a continuous family of quasi-norming bodies, where $G \subset M \backslash \Gamma$ is open and relatively compact in $M$. Assume that $G$ is both $Q$ - and $Q^{d}$-regular. Then

(i) if $W: \bar{G} \rightarrow 2 \mathrm{C}^{n}$ is the Perron envelope of $B(\cdot)$ relative to $P_{Q}^{\mathrm{co}}$, and all the sets $B(\varsigma), \varsigma \in \partial G$, are, in addition, convex, then $z \rightarrow W(z)$ is a continuous family (in the sense of (1.19), (1.20)) of norming bodies, such that $W(\varsigma)=B(\varsigma)$, for $\varsigma \in \partial G$, and the set $\operatorname{gr}(W \mid G)$ is a $P_{Q}^{\text {co }}$-maximum set; and

(ii) if $W: \bar{G} \rightarrow 2^{\mathrm{C}^{n}}$ is the Perron envelope of $B(\cdot)$ relative to $P_{Q}^{\mathrm{psh}}$, then $z \rightarrow$ $W(z)$ is a continuous family of quasi-norming bodies (they are, in particular, polynomially convex), such that $W \mid \partial G=B$, and $\operatorname{gr}(W \mid G)$ is a $P_{Q}^{\mathrm{psh}}$-maximum set.

DEFINITION 2.4. A family of quasi-norming bodies $W: \bar{G} \rightarrow 2 \mathrm{C}^{n}$, which is the Perron envelope of $B(\cdot)$ and satisfies all the requirements of (i) or (ii) will be called the $P_{Q}^{\text {co }}$ - or $P_{Q}^{\text {psh }}$-interpolation family with boundary data $B(\cdot)$. We will use the same terminology for corresponding normed spaces.

A much more general version of Theorem 2.3 is true and will be given in another paper (i.e. for more general classes $Q$ which satisfy a much weaker version of (1.9)(i)). Still, for further reference, we carry some of the arguments under less restrictive assumptions.

PROPOSITION 2.5. Let $M, \Gamma, Q$ be as in Theorem 2.3. Let $G \subset M \backslash \Gamma$ be open, $\bar{G}$ compact in $M$, and $G$ be Q-regular. Let $B: \partial G \rightarrow 2^{\mathrm{C}^{n}}$ be an usc family of compact star-shaped sets. Then

(i) if all the $B(\varsigma)$ 's are convex and $W: \bar{G} \rightarrow 2^{\mathrm{C}^{n}}$ is the Perron envelope of $B(\cdot)$ relative to $P_{Q}^{\text {co }}$, then $z \rightarrow W(z)$ is an usc family of compact convex sets such that $W(\varsigma)=B(\varsigma)$, for $\varsigma \in \partial G$, and $\operatorname{gr}(W \mid G)$ is a $P_{Q}^{c o}$-maximum set;

(ii) if all the $B(\varsigma)$ 's are polynomially convex and $W: \bar{G} \rightarrow 2^{\mathrm{C}^{n}}$ is the Perron envelope of $H(\cdot)$ relative to $P_{Q}^{\mathrm{psh}}$, then $z \rightarrow W(z)$ is an usc family of compact polynomially convex and star-shaped sets, such that $W \mid \partial G=B$ and $\operatorname{gr}(W \mid G)$ is a $P_{Q}^{\mathrm{psh}}$-maximum set; and

(iii) in either case, all $W(z)$ 's are circled, provided all the sets $B(\varsigma)$ are.

PROOF AND REMARKS. (1) In order to prove both the upper semicontinuity of $W(\cdot)$ and compactness of sections $W(z)$, in either (i) or (ii), it suffices to show that 
$\operatorname{gr}(W)=\operatorname{Sat}_{\bar{G} \times \mathbf{C}^{n}}(\operatorname{gr}(B))$ is compact. Observe first, that $\operatorname{Sat}_{\bar{G} \times \mathbf{C}^{n}}(\operatorname{gr}(B))$ is relatively compact. This holds whenever $P$ is a class on $\widetilde{M}=M \times R^{N}$ (here $\mathbf{C}^{n}=R^{2 n}$ ) satisfying (1.13) and the assumptions of [18, Definition 4.11]. (These requirements are much weaker than those of Theorem 2.3.) E.g. the function $p(z, w)=|w|$ belongs to $P$, and if $Z$ is as in Definition 2.1(i), $\max p|Z \leq \max p| \operatorname{gr}(B)=C$, by [18, Corollary 4.4], and so $\operatorname{Sat}_{\bar{G} \times R^{N}}(\operatorname{gr}(B)) \subset\{(z, w): z \in \bar{G},|w| \leq C\}$ and so is relatively compact. Then, by $\left[\mathbf{1 8}\right.$, Corollary 4.12], $\operatorname{Sat}_{\bar{G} \times R^{N}}(\operatorname{gr}(B))=\operatorname{gr} W$ is relatively compact and $\operatorname{gr}(W) \backslash \operatorname{gr}(B)$ is a $P$-maximum set. These conclusions hold, in particular, for $P=P_{Q}^{\text {co }}$ and $P_{Q}^{\text {psh }}$. (Cf. [18, Proposition 1.7] for the relation between $(1.9)$ above and $[\mathbf{1 8},(1.9)]$.)

(2) The statement of (i) and (ii) that $W(\varsigma)=B(\varsigma), \varsigma \in \partial G$, follows immediately from a similar, but more general result [18, Corollary 4.18]. Note that, if $u^{\varsigma} \in$ $Q(\bar{G})$ is a barrier function of Definition 2.2, then the functions $u_{m}^{s}(z, w)=m u(z)$, $(z, w) \in \bar{G} \times \mathbf{C}^{n}$, belong to $P_{Q}^{\text {co }}\left(\bar{G} \times \mathbf{C}^{n}\right) \subset P_{Q}^{\text {psh }}\left(\bar{G} \times \mathbf{C}^{n}\right)$. Clearly, $u_{m}^{s} \mid\{\zeta\} \times \mathbf{C}^{n}=0$ and $\lim \sup _{m} u_{m}^{\varsigma}(z, w)=-\infty$, for $z \neq \varsigma$. Whether $P=P_{Q}^{\text {co }}$ or $P_{Q}^{\text {psh }}$ (in (i) or (ii) respectively), $B(\zeta)$ are $A P$-convex in either case since $A P \mid\{\zeta\} \times \mathbf{C}^{n}$ contains of all convex functions in the first case, and of all plurisubharmonic ones in the second (cf. Definition 1.2 and (1.8)). Thus, assumptions of [18, Corollary 4.18] hold and $W \mid \partial G=B$, as required.

(3) Whether $P=P_{Q}^{\mathrm{co}}$ or $P_{Q}^{\mathrm{psh}}$, the set $\{z\} \times W(z)$ must be $P$-convex. If not, denote the $P$-hull of $\{z\} \times W(z)$ by $Y$; clearly, $Y \backslash\{z\} \times W(z)$ is a $P$-maximum set. (Cf. [21, Theorem 1.6] for a more general fact.) Thus, $Y \subset \operatorname{Sat}_{\bar{G} \times \mathrm{C}^{n}}(\operatorname{gr}(W))$. Since $\operatorname{gr}(W)=\operatorname{Sat}_{\bar{G} \times \mathrm{C}^{n}}(\operatorname{gr}(B))$ and is compact, by [18, Corollary 4.13], it is equal to its own saturation. Hence, $Y \subset \operatorname{gr}(W)$ and so in $\{z\} \times W(z)$, as required.

(4) It is clear, by Definition 2.1(i), that if (1.9)(iv) holds, then $\operatorname{Sat}_{\bar{G} \times \mathrm{C}^{n}}(\operatorname{gr}(B))$ is invariant with respect to rotations $(z, w) \rightarrow\left(z, e^{i \theta} w\right)$, provided $\operatorname{gr}(B)$ is. Thus (iii) follows.

The same argument, applied to homotheties $(z, w) \rightarrow(z, t w), t \in(0,1)$, and using (1.9)(iii), yields star-shapedness in (ii). Q.E.D.

COROLlary 2.6. Let $P=P_{Q}^{\mathrm{co}}$ or $P_{Q}^{\mathrm{psh}}$ on $\widetilde{M}=M \times \mathbf{C}^{n}$, where $M, Q$ are as in Proposition 2.5. Let $G_{1} \subset G \subset M$, where $G_{1}, G$ are open and relatively compact in $M$, and $\varsigma \rightarrow B(\varsigma): \partial G \rightarrow 2^{C^{n}}$ be an usc family of compact, star-shaped sets. If $W: \bar{G} \rightarrow 2^{\mathrm{C}^{n}}$ is the Perron envelope of $B(\cdot)$ of class $P$ and $B_{1}=B \mid \partial G$, then the Perron envelope $W_{1}: \bar{G}_{1} \rightarrow 2^{\mathrm{C}^{n}}$ of $B_{1}(\cdot)$ relative to $P$ is equal to $W \mid \bar{G}$.

ProOF. This fact is actually true whenever $P$ is a class on $\widetilde{M}=M \times R^{N}$, such that part (1) of the last proof works and axioms (1.9)(iii) and [18, (1.8)] hold.

By (1) (of the last proof), $\operatorname{gr}(W)$ and $\operatorname{gr}\left(W_{1}\right)$ are compact and $\operatorname{gr}(W) \backslash \operatorname{gr}(B)$, $\operatorname{gr}\left(W_{1}\right) \backslash \operatorname{gr}\left(B_{1}\right)$ are $P$-maximum sets. Let $Z=\operatorname{gr}(W) \cup \operatorname{gr}\left(W_{1}\right)$. Then $Z$ is compact and $Z \backslash \operatorname{gr}(B)=(\operatorname{gr}(W) \backslash \operatorname{gr}(B)) \cup\left(\operatorname{gr}\left(W_{1}\right) \backslash \operatorname{gr}\left(B_{1}\right)\right)$ is the (locally compact) union of $P$-maximum sets. Hence, $Z \backslash \operatorname{gr}(B)$ is a $P$-maximum set by [18, Proposition 4.9]. By Definition 2.1, $Z \subset \operatorname{gr}(W)$ and $\operatorname{gr}\left(W_{1}\right) \subset \operatorname{gr}\left(W \mid \bar{G}_{1}\right)$. On the other hand, $\operatorname{gr}(\bar{W}) \backslash \operatorname{gr}\left(B_{1}\right)=\operatorname{gr}(W) \cap\left(G_{1} \times R^{N}\right)$ is a $P$-maximum set, cf. [18, Corollary 4.3(i)], and so $\operatorname{gr}\left(W \mid \bar{G}_{1}\right) \subseteq \operatorname{Sat}_{\bar{G}_{1} \times R^{N}}\left(\operatorname{gr}\left(B_{1}\right)\right)=\operatorname{gr}\left(W_{1}\right)$. Thus $W_{1}=W \mid \bar{G}_{1}$. Q.E.D. 
LEMMA 2.7. Let $P$ be a class of usc functions on $\widetilde{M}=M \times R^{N}$ satisfying axioms (1.9)(ii), (iii), (1.11). Let $\varsigma \rightarrow B(\varsigma), \zeta \in \partial G$, be a continuous family of compact, strictly star-shaped neighborhoods of zero in $R^{N}$, where $\bar{G}$ is compact. Let $W: \bar{G} \times 2^{R^{N}}$ be the Perron envelope of $B(\cdot)$ relative to $P$. Assume that $\operatorname{gr}(W)$ is compact. Then all the $W(z)$ 's, $z \in \bar{G}$, are strictly star-shaped neighborhoods of zero.

ProOF. By Proposition 2.5(ii), all the sets $W(z), z \in \bar{G}$, are star-shaped. Observe now that a compact star-shaped set $W \subset R^{N}$ is a strictly star-shaped neighborhood of zero if and only if, for every $t \in(0,1)$, there is an $\varepsilon>0$ such that

$$
\bigcup\left\{t W+x: z \in R^{N},|x| \leq \varepsilon\right\} \subset W .
$$

By assumptions, this relation holds for the sets $B(\varsigma), \varsigma \in \partial G$. Using the continuity of $\varsigma \rightarrow B(\varsigma)$, together with its characterization (1.19), (1.20) and the compactness argument, we find a positive $\varepsilon=\varepsilon(t)$ for every $t \in(0,1)$ so that for every $\varsigma \in \partial G$ :

$$
\bigcup\left\{t B(\varsigma)+x: x \in R^{N},|x| \leq \varepsilon\right\} \subset B(\varsigma) .
$$

We will show now that (2.1) holds for $W=W\left(z_{0}\right), z_{0} \in \bar{G}$, with the same $\varepsilon=\varepsilon(t)$.

Let $w_{0} \in W\left(z_{0}\right)$. By Definition 2.1, there is a compact set $Z \subset \bar{G} \times R^{N}$, such that $Z \backslash \operatorname{gr}(B)$ is a $P$-maximum set and $\left(z_{0}, w_{0}\right) \in Z$. By conditions (1.9)(ii), (iii), if $Z^{t, x}$ denotes the set $\{(z, t w+x):(z, w) \in Z\}$, then $Z^{t, x} \backslash \operatorname{gr}(B)^{t, x}=(Z \backslash \operatorname{gr}(B))^{t, x}$ is a $P$-maximum set. If $t \in(0,1)$ and $|x| \leq \varepsilon=\varepsilon(t)$, then, by $(2.2)$, the set

$$
\left.\bigcup_{|x| \leq \varepsilon} \operatorname{gr}(B)^{t, x}=\bigcup\{\varsigma\} \times(t B(\varsigma)+x):|x| \leq \varepsilon, \varsigma \in \partial G\right\}
$$

is contained in $\operatorname{gr}(B)$, and so, by Definition $2.1, Z^{t, x} \subset \operatorname{gr}(W)$. Hence, $\left(z_{0}, t w_{0}+x\right) \in$ $W\left(z_{0}\right)$ for $|x| \leq \varepsilon$, i.e. $W\left(z_{0}\right)$ is strictly star-shaped. Q.E.D.

LEMMA 2.8. Let $M, \Gamma, Q, G, B, W$ satisfy all the assumptions of Theorem 2.3, except for the $Q$-regularity of $G$. ( $Q^{d}$-regularity is assumed.) Then,

$$
B(\varsigma) \subset \operatorname{Liminf}_{z \rightarrow \varsigma} W(z)
$$

for every $\varsigma \in \partial G$; that is, $W(z)$ is lsc at $\varsigma \in \partial G$ in the sense of (1.20).

Proof. ASSERTION 1. Let $K_{0} \subset \mathbf{C}^{n}$ be a quasi-norming body, $v \in Q^{d}(\bar{G})$ and $K(z)=e^{v(z)} K_{0}, z \in \bar{G}$. Then $\operatorname{gr}(K \mid G)$ is a $P_{Q}^{\text {psh }}$-maximum set. If, in addition, $K_{0}$ is convex, then $\operatorname{gr}(K \mid G)$ is a $P_{Q}^{\text {co }}$-maximum set.

We will show first that the assertion implies the lemma. Fix $\varsigma_{0} \in \partial G$, and $t \in(0,1)$. Let $K_{0}=t B(\varsigma)$ and $v(z)=n u^{\varsigma 0}(z), z \in \bar{G}$, where $u^{\varsigma \circ}$ is the $Q^{d}$-barrier function from Definition 2.2 and $n>0$ is so chosen that

$$
e^{v(\varsigma)} K_{0} \subset \operatorname{Int} B(\varsigma), \quad \varsigma \in \partial G .
$$

By this inclusion, $\operatorname{gr}(K) \backslash \operatorname{gr}(K \mid \partial G)=\operatorname{gr}(K) \backslash \operatorname{gr}(B)$ and, by Assertion 1, this set has local maximum property with respect to functions of class $P$ (where $P=P_{Q}^{\text {co }}$, if $K_{0}$ is convex, and $P=P_{Q}^{\mathrm{psh}}$ otherwise). In addition, $\operatorname{gr}(K)$ is compact, and so $\operatorname{gr}(K) \subseteq \operatorname{gr}(W)$ by Definition 2.1. Since $\lim _{z \rightarrow \varsigma_{0}} v(z)=0$, multifunction $K(z)$ is continuous at $\varsigma_{0}$ in the sense of $(1.19),(1.20)$. Since $K(z) \subset W(z)$ and $t \in(0,1)$ is arbitrary, we conclude that the multifunction $z \rightarrow W(z)$ is lsc at $\zeta_{0}$ in the sense of $(1.20)$. 
PROOF OF ASSERTION 1. Let $p_{0}(w)$ be the Minkowski functional on $\mathbf{C}^{n}$ determined by $K_{0}$ and let $p(z, w)=e^{-v(z)} p_{0}(w), z \in \bar{G}, w \in \mathbf{C}^{n}$. Since $G \times\{0\}$ is a $P$-maximum set (cf. (1.11)) $\operatorname{gr}(K \mid G)$ is a $P$-maximum set, if and only if so is the set $\left\{(z, w) \in G \times \mathbf{C}^{n}: 0<p(z, w) \leq 1\right\}$; cf. [18, Proposition 4.9]. By [18, Corollary 4.16], the latter set is a $P$-maximum set if and only if, for every $\varphi \in C^{(2)}(R)$, with $\varphi^{\prime}(x)>0$ on $R$, the composition $(z, w) \rightarrow \varphi(-\log p(z, w))$ belongs to $P^{d}\left(G \times\left(\mathbf{C}^{n} \backslash\{0\}\right)\right)$. (Note that $p(z, w)$ is positive homogeneous in $w$.) By [20, Theorem 4.9], in order to prove this, it suffices to check that for every $g \in Q$, the function $(z, w) \rightarrow \varphi(g(z)-\log p(z, w))$ is of class $P_{0}^{d}=P_{k+n-1}$ in case $K_{0}$ is polynomially convex, and of class $\left(P_{0}^{\text {co }}\right)^{d}$ if $K_{0}$ is convex. We will prove that the function is $(k+n-1)$-plurisubharmonic in either case, which is enough, because $P_{k+n-1}=P_{0}^{d} \subset\left(P_{0}^{\text {co }}\right)^{d}$. (Note, that $P_{0}^{\text {co }} \subset P_{0} ; P_{0}^{\text {co }}$ consists of plurisubharmonic functions $u(z, w)$, convex in $w$.) We need the following

ASSERTION 2. If $u \in P_{q}(U), U \subset \mathbf{C}^{m}$, and $\varphi:(a, b) \rightarrow R$ is a nondecreasing continuous function with $a<\inf u, \sup u<b$, then $\varphi \circ u \in P_{q+1}(U)$.

Let $u(z, w)=g(z)-\log p(z, w)=(g+v)(z)-\log p_{0}(w)$. By $(1.14),(g+v) \in$ $Q+Q^{d} \subset P_{k-1}(G)$. Since function $-\log p_{0}(w)$ is harmonic on each complex line $(\mathbf{C} \backslash\{0\}) e, e \neq 0, e \in \mathbf{C}^{n}$, clearly, $-\log p_{0} \in P_{n-1}\left(\mathbf{C}^{n} \backslash\{0\}\right)$. Since the functions $(z, w) \rightarrow(g+v)(z)$ and $(z, w) \rightarrow-\log p_{0}(w)$ do not depend on $w$ and $z$, respectively, they belong to classes $P_{k-1}$ and $P_{n-1}$ on $G \times\left(\mathbf{C}^{n} \backslash\{0\}\right)$. (One might use [12, Proposition 1.1] to get this.) By [12, Theorem 5.1], $u \in P_{k-1}+P_{n-1} \subset P_{k+n-2}$. By Assertion 2, $\varphi \circ u \in P_{k+n-1}$, which proves Assertion 1.

ProOF OF ASSERTION 2. Choose first functions $\varphi_{n} \in C^{(2)}(R)$, such that $\varphi_{n}^{\prime}(x)>0$ on $R$ and $\varphi_{n}(x) \searrow \varphi(x), x \in(a, b)$. Clearly, $\varphi_{n}(u(x)) \searrow \varphi(u(x))$, $x \in U$. By Hunt and Murray [3] or [12, Proposition 1.2(vii)], if $\varphi_{n} \circ u$ are $(q+1)$ plurisubharmonic, then so is $\varphi \circ u$. Similarly, by [12, Theorem 2.9], $u$ can be approximated by continuous, $q$-plurisubharmonic functions, so that $u_{n}(x) \searrow u(x)$. If $\varphi$ is continuous, then $\varphi\left(u_{n}(x)\right) \searrow(u(x))$. By these observations, we can assume without loss of generality that $u$ is continuous, $\varphi \in C^{(2)}(R)$ and $\varphi^{\prime}(x)>0$.

Fix $x \in U$. By properties of $\varphi$, there is an $\varepsilon>0$ and two families of increasing affine functions $f_{t}(y)=a_{t} y+b_{t}, g_{t}(y)=c_{t} y+d_{t}, t \in T$, such that $\varphi(y)=$ $\sup _{t}\left[\min \left(f_{t}(y), g_{t}(y)\right)\right]$ for $y \in(u(x)-\varepsilon, u(x)+\varepsilon)$. By the continuity of $u$, we choose a neighborhood $V$ of $x$, such that $\operatorname{diam} u(V)<\varepsilon$. By [12, Propositions 1.2(v), (vi)], $f_{t} \circ u, g_{t} \circ u \in P_{q}(V)$ and by [12, Theorem 6.1], $\min \left(f_{t} \circ u, g_{t} \circ u\right) \in P_{q+1}(V)$. Since $\varphi \circ u$ is continuous and equal to $\sup _{t}\left[\min \left(f_{t} \circ u, g_{t} \circ u\right)\right]$, [12, Proposition 1.2(iv)] implies that $\varphi \circ u \in P_{q+1}(\mathrm{~V})$.

LEMMA 2.9. Let $M, \Gamma$ be as in (1.0), except that $M \backslash \Gamma=K / H$ is a real homogeneous space. Let $P$ be a class of usc functions on $\widetilde{M}=M \times R^{N}$ satisfying conditions (1.9)(i), (iii). Let $G \subset M \backslash \Gamma$ be open and relatively compact in $M, B: \partial G \rightarrow 2^{R^{N}}$ be a continuous family of quasi-norming bodies and let $W: \bar{G} \rightarrow 2^{R^{N}}$ be its Perron envelope relative to $P$. Assume that $z \rightarrow W(z)$ is usc in $\bar{G}$, continuous at each point of $\partial G$ and equal to $B$ on $\partial G$. Then $z \rightarrow W(z)$ is continuous on $\bar{G}$.

ProOF. We apply Walsh's argument [22] to show that $z \rightarrow W(z)$ is lsc at each $z_{0} \in G$. Fix $\varepsilon>0$. By the continuity of $W(\cdot)$ at the points of $\partial G$, we can choose a symmetric neighborhood $K_{0}$ of the unit element of $K$, such that whenever $k \in K_{0}$, 
$\varsigma \in \partial G$ and $t_{k} \varsigma \in \bar{G}$, then

$$
e^{-\varepsilon} W(\varsigma) \subset W\left(t_{k} \varsigma\right) \subset e^{\varepsilon} W(\varsigma)
$$

By properties of homogeneous spaces $\left(z_{0} \in M \backslash \Gamma=K / H\right)$, the set $\left\{t_{k} z_{0}: k \in\right.$ $\left.K_{0}\right\}$ contains a neighborhood $U_{0}$ of $z_{0}$. For every $y \in U_{0}$ define a multifunction $K_{y}: \bar{G} \rightarrow 2^{R^{N}}$ be letting $K_{y}(z)=W(z)$, when $z \in \bar{G} \backslash t_{k} G$, and $W(z) \cup e^{-\varepsilon} W\left(t_{k}^{-1} z\right)$, when $z \in \bar{G} \cap\left(t_{k} G\right)$, where $k=k(y)$ is a fixed element of $K_{0}$, such that $t_{k} y=z_{0}$.

By the upper semicontinuity of the multifunction $z \rightarrow e^{-\varepsilon} W\left(t_{k}^{-1} z\right)$ and (2.4), the correspondence $z \rightarrow K_{y}(z)$ is usc at the points of $\bar{G} \cap t_{k}(\partial G)$ and obviously elsewhere in $\bar{G}$. Thus, $\operatorname{gr}\left(K_{y}\right)$ is compact and equal to $\operatorname{gr}(W) \cup X$, where $X=\left\{\left(z, e^{-\varepsilon} w\right): w \in\right.$ $\left.W\left(t_{k}^{-1} z\right), z \in G \cap\left(t_{k} G\right)\right\}$. By condition (1.9)(i) and (iii), $X$ is a $P$-maximum set as the image, under the map $\left(z^{\prime}, w\right) \rightarrow\left(t_{k}^{-1} z^{\prime}, e^{-\varepsilon} w\right)$, of $\operatorname{gr}\left(W \mid\left(t_{k}^{-1} G\right) \cap G\right)$, which is a $P$-maximum set by part (1) of the proof of Proposition 2.5 .

Note further that, by $(2.4), K_{y}(\varsigma) \subset W(\varsigma)$ for $\zeta \in \partial G$. Hence, $\operatorname{gr}\left(K_{y}\right) \backslash \operatorname{gr}(B)=$ $\operatorname{gr}\left(K_{y} \mid G\right)=\operatorname{gr}(W \mid G) \cup X$. Since $X$ and $\operatorname{gr}(W \mid G)$ are $P$-maximum sets, so is their union, by [12, Proposition 4.9] and, by Definition 2.1, $\operatorname{gr}\left(K_{y}\right) \subset \operatorname{gr}(W)$. Since $K_{y}\left(z_{0}\right)=W\left(z_{0}\right) \cup e^{-\varepsilon} W(y)$, we get $e^{-\varepsilon} W(y) \subset W\left(z_{0}\right)$ for every $y \in U_{0}$. Thus $W(\cdot)$ is lsc at $z_{0}$. Q.E.D.

PROOF OF THEOREM 2.3. The theorem follows immediately by combining Proposition 2.5 and Lemmas 2.7-2.9. Q.E.D.

REMARK 2.10 (REITERATION PROPERTY). In the situation of Theorem 2.3, if $G_{1}$ is open in $G$ and $W_{1}=W\left|\bar{G}_{1}, B_{1}=B\right| \partial G_{1}$, then $z \rightarrow W_{1}(z): \bar{G}_{1} \rightarrow 2 \mathrm{C}^{n}$ is a $P_{Q}^{\text {co }}$ - or $P_{Q}^{\text {psh }}$-interpolation family with boundary data $B_{1}(\cdot)$. (This follows from Theorem 2.3 and Corollary 2.6; cf. Definition 2.4.)

3. Subinterpolation and superinterpolation. The reiteration property (Corollary 2.10) suggests the following extension of the notion of interpolation family to situations when Theorem 2.3 is not applicable.

DEFINITION 3.1. Let $M, \Gamma, Q$ be as in Theorem 2.3 and $X \subset M$ be locally compact, such that $X \subset \bar{D}$, where $D=\operatorname{Int}(X) \backslash \Gamma$. Let $z \rightarrow W(z): X \rightarrow 2^{\mathrm{C}^{n}}$ be a continuous family of quasi-norming bodies. Let $P=P_{Q}^{\text {psh }}$ (or $P_{Q}^{\text {co }}$, if $W(z)$ are convex). Then $W(\cdot)$ is called a $P$-interpolation family on $X$, if for every $G \subset X \backslash \Gamma$, which is open and relatively compact in $X$, and both $Q$-and $Q^{d}$-regular, $W \mid \bar{G}$ is the Perron envelope (relative to the class $P$ ) of the boundary data $W \mid \partial G$.

REMARK 3.2. By Corollary 2.10 and Theorem 2.3, the above definition is consistent with Definition 2.4.

We will show that the notion of an interpolation family has local character. Both for this purpose and for the duality results in $\S 6$, we split this notion into those of subinterpolation and superinterpolation. This approach was initiated by Rochberg $[\mathbf{8}]$ in the context of $[\mathbf{1}, \mathbf{2}]$.

DEFINITION 3.3. Let $M, \Gamma, Q$ satisfy conditions (1.0)-(1.12) and $X \subset M$ be locally compact, such that $X \subset \bar{D}$, where $D=\operatorname{Int}(X) \backslash \Gamma$. Let $z \rightarrow W(z): X \rightarrow 2^{\mathrm{C}^{n}}$ be a family of norming bodies if $P=P_{Q}^{\text {co }}$, or of quasi-norming bodies if $P=$ $P_{Q}^{\text {psh }}$. We say that $W(\cdot)$ is a $P$-superinterpolation family of $X$ (respectively a $P$ subinterpolation family on $X$ ), if $z \rightarrow W(z)$ is lsc in the sense of (1.20) (respectively usc), and for every $G \subset X \backslash \Gamma$, with $G$ open in $M$, relatively compact in $X$ and $Q$ - and $Q^{d}$-regular, and for every $P$-interpolation family $z \rightarrow V(z): \bar{G} \rightarrow 2^{\mathrm{C}^{n}}$, such that 
$W(\varsigma) \subset V(\varsigma), \varsigma \in \partial G$ (respectively $V(\varsigma) \subset W(\varsigma), \varsigma \in \partial G)$ it holds $W(z) \subset V(z)$, $z \in \bar{G}$ (respectively $V(z) \subset W(z), z \in \bar{G})$.

REMARK 3.4. Clearly, $z \rightarrow W(z)$ is a $P$-interpolation family on $X$ if and only if it is both a $P$-subinterpolation and a $P$-superinterpolation family.

THEOREM 3.5. Let $M, \Gamma, Q, P, D, X$ be as in Definition 3.3. Let $z \rightarrow W(z)$ : $X \rightarrow 2^{\mathbf{C}^{n}}$ be an usc family of quasi-norming bodies (or norming ones, if $P=P_{Q}^{\text {co }}$ ). Then $W(\cdot)$ is a $P$-superinterpolation family on $X$, if and only if $\operatorname{gr}(W \mid D)$ is a $P$-maximum set.

LEMMA 3.6. Let $z \rightarrow K(z): X \rightarrow 2^{R^{N}}$ be an usc family of quasi-norming bodies and let $X$ be a compact space. Assume that Minkowski functionals of $K(z)$ belong to a convex cone $S$ of quasi-norms on $R^{N}$. Then there exist continuous multifunctions $K_{n}: X \rightarrow 2^{R^{N}}, n=1,2, \ldots$, such that $K_{n}(z) \supset K_{n+1}(z), \bigcap K_{n}(z)=K(z), z \in X$, $n=1,2, \ldots$, and all the sets $K_{n}(z)$ are quasi-norming bodies whose Minkowski functionals belong to $S$.

PROOF. Since $K(\cdot)$ is usc, for every $\varepsilon>0$ there exists a finite covering $U_{1}, \ldots, U_{m}$ of $X$ and points $z_{j} \in U_{j}, 1 \leq j \leq m$, such that

$$
K(z) \subset e^{\varepsilon} K\left(z_{j}\right) \quad \text { for } z \in U_{j} .
$$

Further, there is a finer covering $V_{1}, V_{2}, \ldots, V_{k}$ and points $v_{i} \in V_{i}, i=1, \ldots, k$, such that for every $i$ the set $\bigcup\left\{V_{s}: V_{s} \cap V_{i} \neq \varnothing\right\}$ is contained in one of the $U_{j}$ 's, and whenever $z \in V_{j}$, then $K(z) \subset e^{\varepsilon} K_{i}$, where $K_{i}:=K\left(v_{i}\right)$. If $p_{z}(\cdot)$ and $p_{i}(\cdot)$ denote the quasi-norms corresponding to $K(z)$ and $K_{i}$ respectively, then

$$
p_{z}(w) \geq e^{-\varepsilon} p_{i}(w), \quad w \in R^{N}, z \in V_{i} .
$$

Choose now a continuous nonnegative decomposition of unity $1 \equiv \varphi_{1}(z)+\cdots+$ $\varphi_{k}(z)$, subordinated to the covering $\left\{V_{i}\right\}$. Let $p_{z}^{\varepsilon}(w)=e^{-2 \varepsilon} \sum_{i=1}^{k} \varphi_{i}(z) p_{i}(w)$. Clearly, $(z, w) \rightarrow p_{z}^{\varepsilon}(w)$ is a continuous function and $p_{z}^{\varepsilon} \in S, z \in X$. By (3.2), $p_{z}(w) \leq e^{-\varepsilon} p_{z}(w), z \in X, w \in R^{N}$.

For an opposite estimate, let now, for given $z, I=\left\{i: z \in V_{i}\right\}$. Then, $p_{z}^{\varepsilon}(w)=$ $\sum_{i \in I} \varphi_{i}(z) p_{i}(w)\left(\right.$ since $\left.\operatorname{supp} \varphi_{i} \subset V_{i}\right)$. Since $\bigcup_{i \in I} V_{i}$ is contained in a certain $U_{j}$, we get, by (3.1), that $K\left(v_{i}\right) \subset e^{\varepsilon} K\left(z_{j}\right), i \in I$, and so $p_{i}(w) \geq e^{-\varepsilon} p_{K\left(z_{j}\right)}(w)$ for $i \in I$, where $p_{K\left(z_{j}\right)}$ denotes the Minkowski functional corresponding to $K\left(z_{j}\right)$. Hence, $p_{z}^{\varepsilon}(w) \geq e^{-3 \varepsilon} p_{K\left(z_{j}\right)}(w)$, seeing that $\sum_{i \in I} \varphi_{i} \equiv 1$.

Let $K_{\varepsilon}(z)$ be the unit ball of $p_{z}^{\varepsilon}$. The above inequalities imply that $e^{\varepsilon} K(z) \subset$ $K_{\varepsilon}(z) \subset e^{3 \varepsilon} K\left(z_{j}\right)$, if $z \in X$ and $z \in U_{j}$. It is now clear that we can choose inductively a sequence $\varepsilon(n) \searrow 0$ and coverings $\left\{U_{j}\right\},\left\{V_{j}\right\}$ etc., so that the resulting sequence $K_{n}(z)=K_{\varepsilon(n)}(z)$ of continuous families of norming bodies satisfies conditions $K_{n+1}(z) \subset \operatorname{Int}\left(K_{n}(z)\right)$ and $\bigcap_{n} K_{n}(z)=K(z), z \in X$. Q.E.D.

REMARK 3.7. Since the classes of norms and of plurisubharmonic quasi-norms form convex cones, the last lemma produces convex $K_{n}(z)$ in case $K(z)$ are convex and polynomially convex strictly star-shaped $K_{n}(z)$ if such are $K(z)$ 's.

PROOF OF THEOREM 3.5. Sufficiency. Let $G \subset D \subset M \backslash \Gamma$ and $V: \bar{G} \rightarrow 2^{\mathrm{C}^{n}}$ be as in Definition 3.3. If $\operatorname{gr}(W \mid D)$ is a $P$-maximum set, then so is its relatively open subset $\operatorname{gr}(W \mid G)$; cf. [18, Corollary 4.3(i)]. Since $\operatorname{gr}(W \mid \bar{G})$ is compact and $W(\varsigma) \subset V(\varsigma)$ for $\varsigma \in \partial G$, by Definition $2.1, \operatorname{gr}(W \mid \bar{G})$ must be contained in the Perron envelope of $V \mid \partial G$, that is $W(z) \varsigma V(z), z \in \bar{G}$, are required. 
Necessity. If $\operatorname{gr}(W \mid D)$ is not a $P$-maximum set, then, by [18, Proposition 4.2(c)], there exist a point $x_{0}=\left(z_{0}, w_{0}\right)$, its neighborhood, say $U_{0} \times B_{0} \subset M \times \mathbf{C}^{n}$, and $f \in P\left(\right.$ nbhd $\left.\bar{U}_{0} \times \bar{B}_{0}\right)$ such that $f\left(x_{0}\right)>f(x)$ for $x \neq x_{0}, x \in \operatorname{gr}(W) \cap$ $\operatorname{nbhd}\left(\bar{U}_{0} \times \bar{B}_{0}\right)$. By adding, if necessary, a constant to $f$, we can assume that $f\left(x_{0}\right)>0>\max f \mid \operatorname{gr}(W) \cap \partial\left(\bar{U}_{0} \times \bar{B}_{0}\right)$.

Now let $g(z, w)=\max (f(z, w), 0)$, whenever $f(z, w)$ is defined, and zero otherwise. By conditions (1.4), (1.7), $g$ is of class $P$ near $\operatorname{gr}\left(W \mid \bar{U}_{0}\right)$; cf. [18, Proposition 3.3]. Note that $\left\{z_{0}\right\} \times W\left(z_{0}\right)$ is a $P$-convex set, i.e. convex with respect to the class $P$; cf. [18, Definition 4.17] and condition (1.8). By a version of the Runge theorem for pseudoconvex classes of functions in [21, Theorem 1.11], there exists $u \in P\left(\bar{U}_{0} \times \mathbf{C}^{n}\right)$, such that $u \equiv g$ near $\left\{z_{0}\right\} \times W\left(z_{0}\right)$. Consequently, there is a neighborhood $G$ of $z_{0}$ and an $\varepsilon>0$, such that $W(z) \subset e^{\varepsilon} W\left(z_{0}\right)$, for $z \in \bar{G}$, and both $u$ and $g$ are defined and equal on $\bar{G} \times e^{\varepsilon} W\left(z_{0}\right)$. In particular,

$$
\begin{gathered}
u\left(z_{0}, w_{0}\right)>u(z, w), \quad(z, w) \neq\left(z_{0}, w_{0}\right), z \in \bar{G}, w \in W(z) . \\
u(\varsigma, w) \leq 0, \quad \varsigma \in \partial G, w \in W(\varsigma) .
\end{gathered}
$$

By the last lemma and remark, there is a continuous family $\varsigma \rightarrow B(\varsigma): \partial G \rightarrow 2^{\mathrm{C}^{n}}$ of $P$-convex quasi-norming bodies, such that $W(\varsigma) \subset B(\varsigma) \subset \operatorname{Int} e^{\varepsilon} W\left(z_{0}\right), \varsigma \in \partial G$, and

$$
\operatorname{gr}(B \mid \partial G) \subset\left\{(\varsigma, w): \varsigma \in \partial G, u(z, w)<\frac{1}{2} u\left(z_{0}, w_{0}\right)\right\} .
$$

(Note that the latter set is open and contains $\operatorname{gr}(W \mid \partial G)$ by (3.3), (3.4).) We can assume without loss of generality that $G$ is strictly pseudoconvex and so both $Q$ - and $Q^{d}$-regular by $(1.8),(1.10)$. By Theorem 2.3 , there is a $P$-interpolation family $z \rightarrow V(z): \bar{G} \rightarrow 2^{\mathrm{C}^{n}}$ with boundary data $B(\varsigma), \varsigma \in \partial G$. Since $W$ is a $P$-superinterpolation family, $W(z) \subset V(z), z \in \bar{G}$.

Observe further that $\operatorname{gr}(V) \subset \bar{G} \times \operatorname{Int}\left(e^{\varepsilon} W\left(z_{0}\right)\right)$. (Indeed, if $p_{0}(w)$ is a Minkowski functional of $e^{\varepsilon} W\left(z_{0}\right)$, the function $p(z, w)=p_{0}(w)$ is of class $P$, and so it has local maximum property on $\operatorname{gr}(V \mid G)$, i.e. $\max p|\operatorname{gr}(V) \leq \max p| \operatorname{gr}(V \mid \partial G)<1$, since $V(\varsigma) \subset \operatorname{Int}\left(e^{\varepsilon} W\left(z_{0}\right)\right), \varsigma \in \partial G$.) Consequently, $u(z, w)$ is of class $P$ near $\operatorname{gr}(V)$. Since $\operatorname{gr}(V) \backslash \operatorname{gr}(V \mid \partial G)$ is a $P$-maximum set (by Theorem 2.3) and $w_{0} \in W\left(z_{0}\right) \subset V\left(z_{0}\right)$, we get $u\left(z_{0}, w_{0}\right) \leq \max u \mid \operatorname{gr}(V \mid \partial G)$ by [18, Corollary 4.4], and the latter number is smaller than $\frac{1}{2} u\left(z_{0}, w_{0}\right)$, by $(3.5)$. This is a contradiction because $u\left(z_{0}, w_{0}\right)>0$ by (3.3), (3.4). Q.E.D.

THEOREM 3.8. Let $M, \Gamma, Q, P, D, X$ be as in Definition 3.3. Let $z \rightarrow W(z)$ : $X \rightarrow 2^{\mathrm{C}^{n}}$ be an lsc family of $P$-convex quasi-norming bodies (where $P=P_{Q}^{\text {co }}$ or $\left.P_{Q}^{\mathrm{psh}}\right)$. Then

(i) $W(\cdot)$ is a P-subinterpolation family on $X$ if and only if the set

$$
Y=\{(z, w): z \in D, w \in \operatorname{Int} W(z)\}
$$

is relatively P-pseudoconvex in $D \times \mathbf{C}^{n} ;$ cf. [18, Definition 5.1(ii)];

(ii) $W(\cdot)$ is a $P$-subinterpolation family on $X$, if and only if there is an open covering $\left\{D_{t}\right\}$ of $D$, such that $W(\cdot)$ is a $P$-subinterpolation family on each $D_{t}$.

PROPOSITION 3.9. If $K, L$ are compact subsets of $\mathbf{C}^{n}$ and $K \subset \operatorname{Int}(L)$, then $\operatorname{hull}(K) \subset \operatorname{Int}($ hull $(L))$, where hull( $(\cdot)$ denotes the polynomial hull.

The proof is very similar to that of Lemma 2.7 and is omitted. 
COROLlaRY 3.10. If $K$ is a quasi-norming body in $\mathbf{C}^{n}$, then so is its polynomial hull.

ProOF. Since $K$ is strictly star-shaped, $t K \subset \operatorname{Int}(K)$ for $t \in(0,1)$ and, by Corollary $3.9, t$ (hull $K)=\operatorname{hull}(t K) \subset \operatorname{Int}(\operatorname{hull}(K))$. Thus hull $(K)$ is strictly starshaped. Other properties of hull $(K)$ are obvious. Q.E.D.

Proof OF THEOREM 3.8. Sufficiency in (i). Let $V: \bar{G} \rightarrow 2^{\mathrm{C}^{n}}$ be as in Definition 3.3; in particular, $G \subset D, \bar{G} \subset X$. Let $Y^{*}=\{(z, w): z \in \bar{G}, w \in$ Int $W(z)\}$. Since $Y^{*} \cap\left(G \times \mathbf{C}^{n}\right)=Y \cap\left(G \times \mathbf{C}^{n}\right)$, it is relatively $P$-pseudoconvex in $G \times \mathbf{C}^{n}$ by [18, Proposition 5.3(a)], and since $G$ is $Q$-regular, by [18, Corollary 5.9] $Y^{*}$ is relatively $P$-pseudoconvex in $\bar{G} \times \mathbf{C}^{n}$. (Note that, if $u^{5}(z)$ is a $Q$ barrier function for $G$ at $\zeta$, then $u_{n}^{\varsigma}(z, w)=n u^{\varsigma}(z)$ are barrier functions of class $P\left(\bar{G} \times \mathbf{C}^{n}\right)$ required in [18, Corollary 5.9].) This and [18, Lemma 5.5] imply that Sat $_{\bar{G} \times \mathbf{C}^{n}}(\operatorname{gr}(t V \mid \partial G)) \subset Y^{*}$ for every $t \in(0,1)$, since $t V(\varsigma) \subset \operatorname{Int} W(\varsigma), \varsigma \in \partial G$, $t \in(0,1)$. Hence, $V(z) \subset W(z), z \in \bar{G}$, as required.

Necessity in (i). If $Y$ is not relatively $P$-pseudoconvex in $G \times \mathbf{C}^{n}$, then, by [18, Theorem 5.7 and Remark 5.8], there are compact sets $K, Z$ and a point $a \in D$, such that $K \subset Y, K \subset Z \subset D \times \mathbf{C}^{n}, Z \subset Y \cup\{a\} \times \mathbf{C}^{n}, Z \cap\{a\} \times \mathbf{C}^{n} \nsubseteq Y$, $a \in \pi(Z)$, where $\pi: M \times \mathbf{C}^{n} \rightarrow M$ denotes the standard projection, and $Z \backslash K$ is a $P$-maximum set. We will show that this is impossible.

Denote $Z(z)=\{w:(z, w) \in Z\} ; K(z)$ is defined similarly. We will choose now a strictly pseudoconvex neighborhood $G$ of $a$, with $\bar{G} \subset D$, and define a multifunction $\varsigma \rightarrow B_{1}(\varsigma): \partial G \rightarrow 2^{\mathrm{C}^{n}}$. If $a \in \pi(K)$, then $K(a) \neq \varnothing$ and $\{a\} \times K(a) \subset Y$. We choose $G$ and a compact neighborhood $K_{0}$ of $K(a)$, so that $K_{0} \subset \operatorname{Int} W(a)$ and $K(z) \subset \operatorname{Int} K_{0}, z \in \bar{G}$. We let $B_{1}(\varsigma)=K_{0} \cup Z(\varsigma), \varsigma \in \partial G$. If $a \in \pi(Z) \backslash \pi(K)$, we choose $G$ so that $\bar{G} \cap \pi(K)=\varnothing$, and define $B_{1}(\varsigma)=\{0\} \cup Z(\varsigma)$, if $\varsigma \in(\partial G) \cap \pi(Z)$, and $\{0\}$ if $\varsigma \in(\partial G) \backslash \pi(Z)$. Note that $B_{1}(\varsigma) \subset \operatorname{Int} W(\varsigma), \varsigma \in \partial G$, since $Z(z) \subset$ int $W(z)$ for $z \neq a$, by the properties of $Z$.

We define now $\varsigma \rightarrow B_{3}(\varsigma): \partial G \rightarrow 2^{\mathrm{C}^{n}}$. If $P=P_{Q}^{\text {co }}$, we let $B_{3}(\varsigma)$ be the circled convex hull of $B_{1}(\varsigma)$. If $P=P_{Q}^{\mathrm{psh}}$, we construct first an usc family of quasinorming bodies $B_{2}(\varsigma), \varsigma \in \partial G$, such that $B_{1}(\varsigma) \subset B_{2}(\varsigma) \subset \operatorname{Int} W(\varsigma), \varsigma \in \partial G$. (Details omitted.) Now let $B_{3}(\varsigma)=$ the polynomial convex hull of $B_{2}(\varsigma)$. Clearly, $\varsigma \rightarrow B_{3}(\varsigma)$ is an usc multifunction and, by Corollary 3.10 , all the $B_{3}(\varsigma)$ 's are quasinorming bodies.

In either case, $\varsigma \rightarrow B_{3}(\varsigma)$ is usc on $\partial G$, all the $B_{3}(\varsigma)$ 's are $P$-convex quasinorming bodies and $\operatorname{gr}\left(B_{3}\right) \subset\{(\varsigma, w): \varsigma \in \partial G, w \in \operatorname{Int} W(\varsigma)\}$. The latter set is open in $\partial G \times \mathbf{C}^{n}$ by (1.20) and, by Lemma 3.6 and Remark 3.7, there is a continuous family $\varsigma \rightarrow B(\varsigma)$, on $\partial G$, of $P$-convex quasi-norming bodies, such that $B_{3}(\varsigma) \subset B(\varsigma) \subset \operatorname{Int} W(\varsigma), \varsigma \in \partial G$. Since $G$ is strictly pseudoconvex, by Theorem 2.3 there is a $P$-interpolation family $V: \bar{G} \rightarrow 2^{\mathrm{C}^{n}}$ with boundary data $B(\cdot)$. Since $W(\cdot)$ is a subinterpolation family and $B(\varsigma) \subset W(\varsigma), \varsigma \in \partial G$; therefore, $V(z) \subset W(z)$ for $z \in \bar{G}$, and so the set $Y^{*}$, defined in the next assertion, is contained in $Y$.

Assertion. If $V: \bar{G} \rightarrow 2^{\mathrm{C}^{n}}$ is a $P$-interpolation family, then the set $Y^{*}=$ $\{(z, w): z \in \bar{G}, w \in \operatorname{Int} W(z)\}$ is relatively $P$-pseudoconvex in $\bar{G} \times \mathbf{C}^{n}$.

The assertion follows directly from Theorem 2.3 and [18, Corollary 5.10].

Denote $K_{1}=\left(\partial G \times \mathbf{C}^{n}\right) \cap Z \cup K \cap\left(\bar{G} \times \mathbf{C}^{n}\right)$ and $Z_{1}=Z \cap\left(\bar{G} \times \mathbf{C}^{n}\right)$. Note that $K_{1} \subset Y^{*}$ (in case $K \cap\left(\bar{G} \times \mathbf{C}^{n}\right) \neq \varnothing$, this intersection is contained in $\bar{G} \times K_{0}$ and 
so in $\left.Y^{*}\right)$. Clearly, $Z_{1} \backslash K_{1}=(Z \backslash K) \cap G \times \mathbf{C}^{n}$ is a $P$-maximum set and so, by the Assertion and [18, Lemma 5.5], $Z_{1} \subset Y^{*}$. Since $Z_{1} \cap\{a\} \times \mathbf{C}^{n}=Z \cap\{a\} \times \mathbf{C}^{n}$, we get $Z \cap\{a\} \times \mathbf{C}^{n} \subset Y$, which is a contradiction. This proves (i).

(ii) Actually, the above proof shows that being a $P$-subinterpolation family is a local property. Otherwise (ii) follows from (i) and [18, Theorem 5.7]. Q.E.D.

REMARK 3.11. Let $M, \Gamma, Q, P, D, X$ be as in Definition 3.3. Let $\left\{G_{t}\right\}_{t \in T}$ be a basis for the topology of $D$ consisting of open sets which are both $Q$ - and $Q^{d}$-regular (e.g. strictly pseudoconvex). Let $z \rightarrow W(z), z \in X$, be a family of $P$-convex, quasinorming bodies in $\mathbf{C}^{n}$. An inspection of the proofs of Theorem 3.5 and 3.8 shows immediately that $W(\cdot)$ is a $P$-subinterpolation (or a $P$-superinterpolation family) on $X$ if and only if conditions of Definition 3.3 are satisfied for $G=G_{t}, t \in T$. In case $M=\mathrm{C}^{k}, G_{t}$ can be Euclidean balls.

COROLlaRY 3.12 (UNIQUENESS PROPERTY). Let $M, \Gamma, Q, P, G$ be as in Theorem 2.3. Let $W: \bar{G} \rightarrow 2^{\mathrm{C}^{n}}$ be a continuous family of $P$-convex quasi-norming bodies. Assume $\left\{G_{t}\right\}_{t \in T}$ is a covering of $G$, such that every $G_{t}$ is both $Q$ - and $Q^{d}$ regular. Assume that $W \mid \bar{G}_{t}$ is the Perron envelope of $W \mid \partial G_{t}$. Then $W: \bar{G} \rightarrow 2^{C^{n}}$ is uniquely determined by its boundary sections $W \mid \partial G$ and is equal to the Perron envelope of $W \mid \partial G$.

Proof. By Theorem 2.3, $W(\cdot)$ is locally a $P$-subinterpolation family, and so it is a subinterpolation family on $\bar{G}$ by Theorem 3.8. By Theorem $2.3, \operatorname{gr}\left(W \mid G_{t}\right)$ is a $P$-maximum set for $t \in T$, and so $\operatorname{gr}(W \mid G)$ is a $P$-maximum set by [18, Corollary 4.3(ii)]. By Theorem 3.5, $W(\cdot)$ is a $P$-superinterpolation family on $\bar{G}$. Eventually, $W$ is a $P$-interpolation family on $\bar{G}$, by Remark 3.4 , and so is the Perron envelope; cf. Remark 3.2. Q.E.D.

4. Properties of interpolating quasi-norms. In this section subinterpolation and superinterpolation families are characterized in terms of corresponding quasi-norms. We use it to the interpolation of operator norm (Corollary 4.5) and to several other applications.

THEOREM 4.1. Let $M, \Gamma, Q, P, X, D$ be as in Definition 3.3. Let $\left\{p_{z}\right\}_{z \in X}$ be quasi-norms on $\mathbf{C}^{n}$. Then, $\left\{\mathbf{C}^{n}, p_{z}(\cdot)\right\}$ form a $P$-subinterpolation family on $X$, if and only if the function $(z, w) \rightarrow \log p_{z}(w)$ is usc on $X \times \mathbf{C}^{n}$ and of class $P_{Q}^{\mathrm{psh}}$ on $D \times \mathbf{C}^{n}$, and the quasi-norms $p_{z}: \mathbf{C}^{n} \rightarrow R$ are plurisubharmonic in case $P=P_{Q}^{\mathrm{psh}}$ and are norms in case $P=P_{Q}^{c o}$. This holds if and only if $p \in P\left(X \times \mathbf{C}^{n}\right)$, where $p(z, w)=p_{z}(w)$.

Proof. Sufficiency. Let $W(z)=\left\{w \in \mathbf{C}^{n}: p_{z}(w) \leq 1\right\}$. Since $p(\cdot, \cdot)$ is usc, $z \rightarrow W(z)$ is lsc by (1.20). The assumptions imply, by $(1.1)(\mathrm{i}),(1.12)$ and $[\mathbf{1 8}$, Proposition 1.14(iv)], that $p=\exp (\log p) \in P\left(X \times \mathbf{C}^{n}\right)$. By [18, Proposition 5.8(e)], the set $Y=\left\{(z, w) \in D \times \mathbf{C}^{n}: p(z, w)<1\right\}$, which is equal to the set (3.6), is $P$-pseudoconvex, and so $z \rightarrow W(z)$ is a $P$-subinterpolation family by Theorem 3.8 .

Observe further that, if $p \in P\left(X \times \mathbf{C}^{n}\right)$, then $\log p \in P_{Q}^{\mathrm{psh}}$. Indeed, by result of $[20, \S 5]$,

if $u \in Q(U), U \subset M$, and $|h| u \in Q(U)$ for every function $h(z)$, holomorphic and nonvanishing on $U$, then $\log u \in Q(U)$. 
By Definition 1.1, we have to check that $\log u(z)=\log p(z, F(z))$ is of class $Q$, whenever $F$ is analytic. However, for every analytic function $h(z)$, the product $|h(z)| u(z)=p(z, h(z) F(z))$ is of class $Q$. Thus $u \in Q$, by the last reference.

Necessity. If $z \rightarrow W(z)$ is an lsc family on $X$, then $p(z, w)$ is usc on $X \times \mathbf{C}^{n}$ by (1.20). In order to observe that $\log p \mid D \times \mathbf{C}^{n} \in P_{Q}^{\mathrm{psh}}$, i.e. that the function $z \rightarrow \log p(z, F(z))$ is of class $Q$, where $F(z)$ is as in Definition 1.1, it suffices to show, by (1.15) and [18, Definition 1.11 and Lemma 2.8], that for every $v \in Q^{d}(\mathrm{nbhd} \bar{G})$, it holds that

$$
\max _{z \in \bar{G}}(v(z)+\log p(z, F(z))) \leq \max _{\zeta \in \partial G}(v(\varsigma)+\log p(\varsigma, F(\varsigma))),
$$

where the sets $G$ form a basis of strictly pseudoconvex neighborhoods for the topology of $D$. We consider $G$ small enough so that, for given $F \in \operatorname{Hol}\left(\bar{G}, \mathbf{C}^{n}\right)$, there exist $F_{1}, \ldots, F_{n-1} \in \operatorname{Hol}\left(\bar{G}, \mathbf{C}^{n}\right)$ such that, for every $z \in \bar{G}$, the vectors $F(z), F_{1}(z), \ldots, F_{n-1}(z)$, form a basis of $\mathbf{C}^{n}$. Now let $L(z)=\{\alpha F(z): \alpha \in \mathbf{C}$, $\left.|\alpha| \leq e^{v(z)}\right\}, z \in \bar{G}$, and let

$$
K_{\varepsilon}(z)=\left\{e^{v(z)} w: w \in \mathbf{C}^{n},\left|w_{1}\right|^{2}+\varepsilon^{-2}\left(\left|w_{2}\right|^{2}+\cdots+\left|w_{n}\right|^{2}\right) \leq 1\right\}
$$

for $z \in \bar{G}, \varepsilon>0$. By Assertion 1 in the proof of Lemma 2.8, $\operatorname{gr}\left(K_{\varepsilon} \mid G\right)$ is a $P$ maximum set. Let $L_{\varepsilon}: \bar{G} \rightarrow 2^{\mathrm{C}^{n}}$ be the set-valued function whose graph is the image of $\operatorname{gr}\left(K_{\varepsilon}\right)$ under the biholomorphic map $(z, w) \rightarrow\left(z, w_{1} F(z)+w_{2} F_{1}(z)+\right.$ $\left.\cdots+w_{n} F_{n-1}(z)\right)$. The class $P$ is invariant with respect to such maps (cf. Definition 1.1 or [20, Proposition 4.4], and so $\operatorname{gr}\left(L_{\varepsilon} \mid G\right)$ is a $P$-maximum set.

Note now that, by the homogenity of $p_{z}(w)$ and (1.5), the inequality (4.2) is equivalent to the following property:

$$
\text { whenever } \max _{\varsigma \in \partial G} p\left(\varsigma, e^{v(\varsigma)} F(\varsigma)\right)<1 \text {, then } \max _{z \in \bar{G}} p\left(z, e^{v(z)} F(z)\right) \leq 1 \text {. }
$$

The first condition means that $\operatorname{gr}(L \mid \partial G) \subset Y$; cf. (3.6). Seeing that

$$
\bigcap_{\varepsilon>0} \operatorname{gr}\left(L_{\varepsilon} \mid \partial G\right)=\operatorname{gr}(L \mid \partial G)
$$

$L_{\varepsilon}$ forms a decreasing family and $Y$ is open, we can conclude that $\operatorname{gr}\left(L_{\varepsilon} \mid \partial G\right) \subset Y$ for some $\varepsilon>0$, i.e. $L_{\varepsilon}(\varsigma) \subset W(\varsigma), \varsigma \in \partial G$. Since $L_{\varepsilon}(\varsigma), \varsigma \in \partial G$, is a continuous family of norming bodies, by Theorem 2.3 , there is a $P$-interpolation family $V: \bar{G} \rightarrow$ $2^{\mathrm{C}^{n}}$, such that $V \mid \partial G=L_{\varepsilon}$. Consequently, $V(z) \subset W(z), z \in \bar{G}$, because $W(\cdot)$ is a subinterpolation family, and $L_{\varepsilon}(z) \subset V(z), z \in \bar{G}$, since $\operatorname{gr}\left(L_{\varepsilon} \mid G\right)$ is a $P$-maximum set. Hence, $L(z) \subset W(z), z \in \bar{G}$, i.e. $p\left(z, e^{v(z)} F(z)\right) \leq 1, z \in \bar{G}$. Q.E.D.

Note that the last theorem gives a new way to obtain Theorem 3.8(ii).

COROLlaRY 4.2. Let $M, \Gamma, Q, G$ be as in Theorem 2.3. Let $\left\{r_{s}\right\}_{s \in \partial G}$ be a continuous family of norms (or plurisubharmonic quasi-norms) on $\mathbf{C}^{n}$. Then there is the greatest function $p \in \operatorname{usc}\left(\bar{G} \times \mathbf{C}^{n}\right)$ with the following properties:

(i) $p(\varsigma, w) \leq r_{\varsigma}(w),(\varsigma, w) \in \partial G \times \mathbf{C}^{n}$;

(ii) $p \mid G \times \mathbf{C}^{n} \in P_{Q}^{\text {co }}\left(G \times \mathbf{C}^{n}\right)$ in case $r_{\varsigma}$ are norms, and $p \mid G \times \mathbf{C}^{n} \in P_{Q}^{\mathrm{psh}}\left(G \times \mathbf{C}^{n}\right)$ otherwise. In addition, the extremal function $p$ is continuous on $\bar{G} \times \mathbf{C}^{n}$ and $z \rightarrow$ $W(z), W(z)=\{w: p(z, w) \leq 1\}$, is an interpolation family of class $P_{Q}^{\mathrm{co}}$ or $P_{Q}^{\mathrm{psh}}$, respectively. 
Proof. As usual, let $P=P_{Q}^{\text {co }}$ or $P_{Q}^{\text {psh }}$. Let $B(\varsigma)=\left\{w: r_{\varsigma}(w) \leq 1\right\}$ and let $W^{*}(z)$ be the $P$-interpolation family in $\bar{G}$, determined by the boundary data $B(\varsigma)$, $\varsigma \in \partial G$, which exists by Theorem 2.3. Let $p_{z}^{*}$ denote the Minkowski functional of $W^{*}(z)$. Then $p_{\varsigma}^{*}=r_{\varsigma}$ and $p^{*}(z, w)=p_{z}^{*}(w)$ is continuous on $\bar{G} \times \mathbf{C}^{n}$, by Theorem 2.3 , and $p^{*} \in P\left(G \times \mathbf{C}^{n}\right)$, by Theorem 4.1. Thus $p^{*}(z, w)$ satisfies conditions (i), (ii), and it suffices to show that $p^{*}(z, w) \geq p_{1}(z, w)$ if $p_{1}$ is any other function satisfying these conditions.

To see this, let $V(z)=\left\{w \in \mathbf{C}^{n}: p_{1}(z, w) \leq 1\right\}$. By Theorem 4.1, $z \rightarrow V(z)$ is a $P$-subinterpolation family in $\bar{G}$ and, by (i), $B(\varsigma) \subset V(\varsigma)$. Since $W^{*}(\cdot)$ is an interpolation family, $W^{*}(z) \subset V(z), z \in \bar{G}$, and so $p_{z}^{*}(w) \geq p_{1}(z, w), z \in G$, $w \in \mathbf{C}^{n}$. Q.E.D.

COROLlary 4.3. Let $M, \Gamma, Q, P, X, D$ be as in Definition 3.3 and let $p_{z}, z \in X$, be quasi-norms on $\mathbf{C}^{n}$. Denote $u(z, w)=\max \left(0, \log p_{z}(w)\right)$. Then

(i) $\left\{\mathbf{C}^{n}, p_{z}\right\}_{z \in X}$ is a P-interpolation family if and only if both $u \in P\left(X \times \mathbf{C}^{n}\right)$ and $(-u) \in P^{d}\left(X \times\left(\mathbf{C}^{n} \backslash\{0\}\right)\right)$;

(ii) $\left\{\mathbf{C}^{n}, p_{z}\right\}_{z \in X}$ is a P-superinterpolation family if and only if $(-u) \in$ $P^{d}\left(X \times\left(\mathbf{C}^{n} \backslash\{0\}\right)\right) ;$ and

(iii) (ii) holds if and only if, for every $\varphi \in C^{(2)}(R)$ with $\varphi^{1}(x)>0, x \in R$, the function $\varphi\left(-\log p_{z}(w)\right)$ belongs to $P^{d}\left(G \times\left(\mathbf{C}^{n} \backslash\{0\}\right)\right)$.

ProOF. Parts (ii) and (iii) follow directly from Theorem 3.5 and [18, Corollary 4.16]. Condition (i) is necessary by Theorem 4.1 and part (ii). By the same facts, in order to conclude that (i) is sufficient, we have to check that $\log p \in P_{Q}^{\mathrm{psh}}$. This is true, because $u(z, n w)-\log n=\max \left(\log p_{z}(w),-n\right)$ and so $u(z, n w)-$ $\log n \searrow \log p(z, w)$, where $u(z, n w)-\log n$ is of class $P_{Q}^{\text {psh }}$ by conditions (1.5) and (1.9)(iii). Q.E.D.

COROllary 4.4. Let $M, \Gamma, Q, X, D$ be as in Definition 3.3. Let $\left\{\mathbf{C}^{n}, p_{z}\right\}_{z \in X}$ be a $P_{Q}^{\mathrm{co}}$-superinterpolation family of normed spaces. Then, the dual normed space form a $P_{Q^{d}}^{\text {co }}$-subinterpolation family.

ProOF. Let $q_{z}$ denote the dual norm to $p_{z}, z \in X$. Then

$$
q_{z}(\xi)=\max \{|\langle w, \xi\rangle|: w \in W(z)\}, \quad z \in X, \xi \in \mathbf{C}^{n},
$$

where $\langle w, \xi\rangle=w_{1} \xi_{1}+\cdots+w_{n} \xi_{n}$, and $W(z)=\left\{w: p_{z}(w) \leq 1\right\}$. Since $z \rightarrow W(z)$ is usc and compact valued, the function $q(z, \xi)=q_{z}(\xi)$ is obviously usc on $X \times \mathbf{C}^{n}$. By Theorem 4.1, we have to show that $\log q \in P_{Q^{d}}^{\mathrm{psh}}\left(D \times \mathbf{C}^{n}\right)$, which, by Definition 1.1 and [18, Definition 1.11], reduces to checking that: the function $v(z)=f(z)+$ $\log q(z, F(z))$ has local maximum property in $G_{0} \subset D$, provided $f \in Q\left(G_{0}\right)$ and $F: G_{0} \rightarrow \mathbf{C}^{n}$ is holomorphic. (Note that $w \rightarrow \log q_{z}(w)$ is plurisubharmonic for each $z \in G_{0}$.)

Observe that $v(z)=\max \{u(z, w): w \in W(z)\}, z \in G_{0}$, where $u(z, w)=$ $f(z)+\log |\langle w, F(z)\rangle|$. Clearly, $u \in P_{Q}^{\mathrm{psh}}$ and so, by Proposition 1.3 (with $s=0$ ), $v \in P_{k-1}\left(G_{0}\right)$, and so has the local maximum property by $[\mathbf{4}$, or 12, Proposition 1.2(viii)]. Q.E.D.

Corollary 4.5. Let $M, \Gamma, Q, P, X, D$ be as in Definition 3.3. Assume that $\left\{\mathbf{C}^{n}, p_{z}\right\}_{z \in X}$ form a $P$-superinterpolation family of normed or quasi-normed spaces 
and $\left\{\mathbf{C}^{m}, q_{z}\right\}_{z \in X}$ form a P-subinterpolation family of normed or quasi-normed spaces. Let $z \rightarrow T_{z}: D \rightarrow L\left(\mathbf{C}^{n}, \mathbf{C}^{m}\right)$ be an analytic family of linear transformations and $r\left(T_{z}\right)=\sup \left\{q_{z}\left(T_{z} w\right) / p_{z}(w): w \in \mathbf{C}^{n} \backslash\{0\}\right\}$. Then, the function $z \rightarrow \log r\left(T_{z}\right)$ has local maximum property in D. More precisely, it belongs to $(A Q)^{d}$ and is, in particular, $(k-1)$-plurisubharmic.

Proof. Case $P=P_{Q}^{\text {psh }}$. Since $\log r(z)=\max \left\{\log q\left(z, T_{z} w\right): w \in W(z)\right\}$, where $W(z)=\left\{w \in \mathbf{C}^{n}: p(z, w) \leq 1\right\}, \log q \in P_{Q}^{\mathrm{psh}}$ by Theorem 4.1 and $\operatorname{gr}(W)$ is a $P_{Q}^{\mathrm{psh}}$-maximum set, we conclude, by Proposition 1.3 , that $\log r \in(A Q)^{d} \subset$ $P_{k-1}$ (cf. Definition 1.2). If $P=P_{Q}^{\text {co }}$, we apply the same argument to $r(z)=$ $\max \left\{q\left(z, T_{z}(w)\right): w \in W(z)\right\}$ and get $r \in(A Q)^{d}(D) \subset P_{k-1}(D)$. Replacing $T_{z}(w)$ by $h(z) T_{z}(w)$, where $h(z)$ is analytic and $\neq 0$, we obtain that $|h(z)| r(z)$ is $(k-1)$ plurisubharmonic for every such $h(\cdot)$ and, by (4.2), $\log r$ is $(k-1)$-plurisubharmonic (more precisely of class $\left.(A Q)^{d}(D)\right)$. Q.E.D.

REMARK 4.6. The quasi-norm $r\left(T_{z}\right)$ (norm, in case $q_{z}$ are norms) has a natural meaning also when $T_{z}: \mathbf{C}^{n} \rightarrow \mathbf{C}^{m}$ are complex-homogeneous polynomial mappings of constant degree and Corollaries 4.4 and 4.5 extend to this context. We will now show that the regularity assumption on $G$ in Corollary 3.12 can be omitted.

COROllary 4.7. Let $M, \Gamma, Q$ be as in Theorem 2.3. Let $G \subset M \backslash \Gamma$ be open, with compact closure in $M$, and $p_{z}, z \in \bar{G}$, be a continuous family of norms (respectively quasi-norms) on $\mathbf{C}^{n}$. Assume that $\left\{\mathbf{C}^{n}, p_{z}\right\}_{z \in G}$ form locally a $P_{Q}^{\text {co }}$ (respectively $P_{Q}^{\mathrm{psh}}$-) interpolation family. Then $\left\{\mathbf{C}^{n}, p_{z}\right\}_{z \in \bar{G}}$ form an interpolation family on $\bar{G}$ and are uniquely determined by the boundary data $p_{\varsigma}, \varsigma \in \partial G$.

PROOF. The first part of the statement is already well known; it follows also from Corollary 4.3(i). The uniqueness is a direct consequence of the next lemma.

LEMMA 4.8. Let $M, \Gamma, Q$ be as in Theorem 2.3. Let $G \subset M \backslash \Gamma$ be open and relatively compact in $M$. Let $\{W(z)\}_{z \in \bar{G}}$ and $\{V(z)\}_{z \in \bar{G}}$ be P-superinterpolation and $P$-subinterpolation families of quasi-norming bodies, respectively (where $P=$ $P_{Q}^{\text {co }}$ or $\left.P_{Q}^{\mathrm{psh}}\right)$. Assume that $W(\varsigma) \subset V(\varsigma), \varsigma \in \partial G$. Then, $W(z) \subset V(z)$ for $z \in \bar{G}$.

ProOF. Let $r(z)$ denote the quasi-norm of the identity transformation

$$
I:\left(\mathbf{C}^{n}, p_{W(z)}\right) \rightarrow\left(\mathbf{C}^{n}, p_{V(z)}\right),
$$

where $p_{W(z)}, p_{V(z)}$ denote the corresponding Minkowski quasi-norms. Since $W(\varsigma) \subset$ $V(\varsigma), \zeta \in \partial G, \log r(\varsigma) \leq 0$ and $\partial G$ and, by the local maximum property of $\log r$, established in Corollary 4.5, $\log r(z) \leq 0$ for $z \in G$, i.e. $W(z) \subset V(z), z \in G$. Q.E.D.

REMARK 4.9. It can be now shown easily that, if we have two interpolation families on domains $G$ and $H$, respectively, which agree on $(\partial G) \cap \bar{H}$ and $(\partial H) \cap \bar{G}$, then they define one interpolation family on $\bar{G} \cup \bar{H}$, which is an analog of a result of $\mathrm{T}$. Wolff $[\mathbf{2 3}]$. We omit further details.

5. A minimum principle for plurisubharmonic functions. The next result is our crucial tool in the proof of the duality theorem in $\S 6$. It was inspired by Kiselman [4, Theorem 2.2]. 
THEOREM 5.1. Let $u(z, w)$ be a plurisubharmonic function on $U \times \mathbf{C}^{q}$, such that slice functions $w \rightarrow u(z, w)$ are convex for $z \in U$, where $U$ is a complex manifold. Then, the function $v(z)=\inf \left\{u(z, w): w \in \mathbf{C}^{q}\right\}$ is q-plurisubharmonic in $U$.

(a) If, in addition, $u$ is strictly plurisubharmonic in $(z, w)$ and strongly convex in $w$ (i.e. real Hessian of $w \rightarrow u(z, w)$ is positive definite), and $\lim _{|w| \rightarrow \infty} u(z, w)=$ $+\infty$, then $v(z)$ is strictly q-plurisubharmonic.

The next lemma is presumably well known; cf. Kiselman [5, Theorem 2.2] for the one-dimensional version.

LEMMA 5.2. Let $u(x, y)$ be a $C^{(2)}$-smooth function on $D \times R^{N}$, where $D$ is open in $R^{K}$, such that for every $x \in D$, the slice function $y \rightarrow u(x, y)$ is strongly convex and $\lim _{|y| \rightarrow \infty} u(x, y)=\infty$. Let $v(x)=\inf \left\{u(x, y): y \in R^{N}\right\}, x \in D$. Then $v$ is $C^{(2)}$-smooth and the Hessian matrix of $v$ at $a$ is equal to

$$
\text { Hess } v(a)=H_{11}-H_{12} H_{22}^{-1} H_{21}, \quad a \in D,
$$

where $\left(x, H_{11} x\right)+\left(y, H_{12} x\right)+\left(x, H_{21} y\right)+\left(y, H_{22} y\right)$ is the Hessian form of $u$ at $(a, b)$, where $u(a, b)=v(a)$.

ProOF (SKeTCH). By the assumptions, for every $x \in D$, the function $y \rightarrow$ $u(x, y)$ has strict global minimum at a unique point $y=\Phi(x)$. Let $f(x, y) \in R^{N}$ denote the gradient of $y \rightarrow u(x, y)$ at $(x, y)$ and let $d_{2} f(x, y)$ denote the differential of the map $y \rightarrow f(x, y)$ at $y$. The strong convexity assumption implies that $y=\Phi(x)$ is the unique solution of $f(x, y)=0$ and $d_{2} f(x, \Phi(x))$ is an isomorphism, $x \in D$. By the implicit function theorem [7, Corollary 1.3.9 and Lemma 1.3.7], $\Phi: D \rightarrow R^{N}$ is $C^{(1)}$-smooth and

$$
(d \Phi)(a)=-H_{22}^{-1} H_{21}
$$

(since $\left(d_{2} f\right)(a, \Phi(a))=H_{22}$ and $\left.\left(d_{1} f\right)(a, \Phi(a))=H_{21}\right)$.

Since $v(x)=u(x, \Phi(x)), x \in D, v$ is $C^{(1)}$-smooth. Furthermore, $d v(x)=$ $\left(d_{1} u\right)(x, \Phi(x))$ (note, that $d_{2} u(x, \Phi(x))=f(x, \Phi(x))=0$ ) and so, $v \in C^{(2)}(D)$. Differentiating the last identity and using (5.2), we get Hess $v(a)=H_{11}+H_{12} \circ d \Phi(a)=$ $H_{11}-H_{12} H_{22}^{-1} H_{11}$. Q.E.D.

The next fact is a generalization of Kiselman [4, Theorem 2.2] to smooth $q$ plurisubharmonic functions. (It is false in the nonsmooth case.)

LEMMA 5.3. Let $u(z, w)$ be $C^{(2)}$-smooth and q-plurisubharmonic on $D \times \mathbf{C}^{n}$, where $D$ is open in $\mathbf{C}^{k}$. Assume that $w \rightarrow u(z, w)$ is a convex function for each $z \in D$ and $u(z, w)$ does not depend on $\operatorname{Im} w=\left(\operatorname{Im} w_{1}, \ldots, \operatorname{Im} w_{n}\right)$. Then $v(z)=$ $\inf \left\{u(z, w): w \in \mathbf{C}^{n}\right\}$ is q-plurisubharmonic in $D$.

(a) If, in addition, $u(z, w)$ is strictly q-plurisubharmonic in $(z, w)$, strongly convex in $\operatorname{Re} w$ and $u(z, w) \rightarrow \infty$ as $|\operatorname{Re} w| \rightarrow \infty$, then $v(z)$ is strictly q-plurisubharmonic.

(Recall that a function is strictly $q$-plurisubharmonic if it is $C^{(2)}$-smooth and its complex Hessian at every point has not more than $q$ nonpositive eigenvalues.)

PROOF. Write $w_{j}=t_{j}+i s_{j}, j=1, \ldots, n$. To reduce the proof to the case (a), let $u_{\varepsilon}(z, t, s)=u(z, t, s)+\varepsilon\left(|z|^{2}+|t|^{2}\right)$ and let $v_{\varepsilon}(z)=\inf u_{\varepsilon}(z, t, s), \varepsilon>0$. Clearly, functions $u_{\varepsilon}(z, t, s)$ have all the properties required in (a), and so $v_{\varepsilon} \in P_{q}(D)$ 
(assuming (a)). Since $v_{\varepsilon}(z) \backslash v(z), z \in D, v$ must belong to $P_{q}(D)$ by [3]; cf. also [12, Proposition 1.2(vii)].

We will now prove case (a) by induction, using the following assertion, whose proof will be given later.

Assertion. (a) is true for $n=1$.

Let $v_{r}\left(z, w_{1}, \ldots, w_{n-r}\right)=\inf \left\{u\left(z, w_{1}, \ldots, w_{n}\right):\left(w_{n-r+1}, \ldots, w_{n}\right) \in \mathbf{C}^{r}\right\}$. Since $v_{r}\left(z, w_{1}, \ldots, w_{n-r}\right)=\inf \left\{u(z, t, s):\left(t_{n-r+1}, \ldots, t_{n}\right) \in R^{r}\right\}$ and $u(z, w)$ is $C^{(2)}$-smooth and strongly convex in $\left(t_{n-r+1}, \ldots, t_{n}\right)$, we conclude that $v_{r} \in$ $C^{(2)}\left(D \times \mathbf{C}^{r}\right)$. Furthermore, $v_{r}\left(z, t_{1}, \ldots, t_{n-r}, s_{1}, \ldots, s_{n-r}\right)$ is strongly convex in $t_{1}, \ldots, t_{n-r}$, which follows by applying formula (5.1) to the real Hessian form of $t \rightarrow v(z, t, s)$ split into $\left(H_{11} t^{\prime}, t^{\prime}\right)+\left(t^{\prime \prime}, H_{12} t^{\prime}\right)+\left(t^{\prime}, H_{21} t^{\prime \prime}\right)+\left(t^{\prime \prime}, H_{22} t^{\prime \prime}\right)$, with $t^{\prime} \in R^{n-r}, t^{\prime \prime} \in R^{r}$. (Clearly, if the latter form is positive definite, then so is $H_{11}-H_{12} H_{22}^{-1} H_{21}$.) Obviously, $v_{r}$ does not depend on $\left(s_{n-r+1}, \ldots, s_{n}\right)=$ $\operatorname{Im}\left(w_{n-r+1}, \ldots, w_{n}\right)$ and $v_{r}\left(z, t_{1}, \ldots, t_{n-r}, s_{1}, \ldots, s_{n-r}\right) \rightarrow \infty$, as $\left|\left(t_{1}, \ldots, t_{n-r}\right)\right| \rightarrow$ $\infty$.

Altogether, each $v_{r}, 0 \leq r \leq n$, satisfies all the assumptions of the assertion and $v_{r+1}\left(z, w_{1}, \ldots, w_{n-r-1}\right)=\inf \left\{v_{r}\left(z, w_{1}, \ldots, w_{n-r}\right): w_{n-r} \in \mathbf{C}\right\}$, where $v_{0}=u$ and $v_{n}=v$. Applying the assertion inductively to pairs $v_{r}, v_{r+1}$, we obtain that $v$ is strictly $q$-plurisubharmonic, as required.

It remains to prove the assertion. Clearly, it is enough to show that $v \mid P$ is strictly $q$-plurisubharmonic for every $(q+1)$-dimensional complex plane $P \subset \mathbf{C}^{k}$. Observe further, that if $v_{1}=v \mid P$, then $v_{1}(z)=\inf _{w \in \mathrm{C}} u_{1}(z, w)$, where $u_{1}=$ $u \mid P \times \mathbf{C}, z \in P \cap D$. The function $u_{1}(z, w)$ is strictly $q$-plurisubharmonic in $(P \cap D) \times \mathbf{C}$ and satisfies all the remaining assumptions of the assertion. Hence, it suffices to prove the assertion in the case $P=\mathbf{C}^{n}$, i.e. $n=q+1$.

When $n=q+1$, we have to show that the complex Hessian, denoted Hess ${ }^{\mathbf{C}} v(a)$, has at least one positive eigenvalue for every $a \in D$. By Lemma 5.2 and (5.1), Hess ${ }^{\mathrm{C}} v(a)$ depends only on second-order terms of the Taylor polynomial at $z=a$. So, we can assume without loss of generality that $a=0$ and $u$ is a homogeneous quadratic polynomial, i.e.

$$
u(z, w)=\langle\bar{z}, A z\rangle+2 \operatorname{Re}\langle\bar{L}, z\rangle t+c^{2}+\langle z, B z\rangle,
$$

where $\langle\cdot, \cdot\rangle$ stands for the $\mathbf{C}$-bilinear product, $A$ is a Hermitian matrix, $B$ is a symmetric matrix, $c>0$ and $t=\operatorname{Re} w$. Since the pluriharmonic term $\langle z, B z\rangle$ influence neither Hess ${ }^{\mathbf{C}} u(0)$ nor Hess ${ }^{\mathbf{C}} v(0)$, we will omit it. Then, $v(z)=\min _{t} u(z, t, s)=$ $\langle\bar{z}, A z\rangle-C^{-2}(\operatorname{Re}\langle\bar{L}, z\rangle)^{2}=\langle\bar{z}, A z\rangle-\frac{1}{2} c^{-2}\langle\bar{L}, z\rangle\langle L, \bar{z}\rangle+$ pluriharmonic terms in $z$. Hence, Hess ${ }^{\mathrm{C}} v(0)=A-\frac{1}{2} c^{-2} L^{T} L^{-}$. On the other hand, substituting $t=\frac{1}{2}(w+\bar{w})$ into (5.3), we get: $u(z, w)=\langle\bar{z}, A z\rangle+\frac{1}{2}\langle\bar{L}, z\rangle \bar{w}+\frac{1}{2}\langle L, \bar{z}\rangle w+\frac{1}{2} c^{2} \bar{w} w+$ pluriharmonic terms. By this, Hess ${ }^{\mathrm{C}} u(0)=V+X^{T} X$, where $V$ is the $(n+1) \times(n+1)$ matrix, with the $n \times n$ top left block equal to Hess ${ }^{\mathrm{C}} v(0)$ and other terms zero, and $X^{t}=\left[2^{-1 / 2} c^{-1} L^{T}, 2^{-1 / 2} c\right], X \in \mathbf{C}^{n+1}$.

Suppose that Hess ${ }^{\mathrm{C}} v(0)$ is negative semidefinite, i.e. $(-V)$ is positive semidefinite. Then, the matrix $(-V)+\left(\operatorname{Hess}^{\mathrm{C}} u\right)(0)=X^{T} X$ has at least two positive eigenvalues, because $\operatorname{Hess}^{\mathrm{C}} u(0)$ does (by assumption). This is a contradiction, for $X^{T} X$ has precisely one positive eigenvalue. Q.E.D.

PROOF OF THEOREM 5.1. We prove part (a) first. In order to apply Lemma 5.3 , we transform $u$ into a function $U$, which is constant in certain imaginary 
directions, by adding more variables. Since the problem is local in the $z$ variable, we assume that $U$ is an open subset of $\mathbf{C}^{k}$.

Define $T: \mathbf{C}^{q} \times \mathbf{C}^{q} \rightarrow \mathbf{C}^{q}$ by letting $T\left(\xi_{1}, \ldots, \xi_{n}, \varsigma_{1}, \ldots, \varsigma_{n}\right)=\left(\operatorname{Re} \xi_{1}+i \operatorname{Re} \varsigma_{1}, \ldots\right.$, $\left.\operatorname{Re} \xi_{q}+i \operatorname{Re} \varsigma_{q}\right)$ and let $U(z, \xi, \varsigma)=u(z, T(\xi, \varsigma))$ for $(z, \xi, \varsigma) \in U \times \mathbf{C}^{2 q}$. Clearly, if $u(z, w)$ satisfies the assumptions of Theorem $5.1(\mathrm{a})$, then $U(z, \xi, \zeta)$ is smooth, does not depend on $\operatorname{Im} \xi$ and $\operatorname{Im} \varsigma$, is convex in $(\xi, \varsigma)$ and strongly convex in $(\operatorname{Re} \xi, \operatorname{Re} \zeta)$, and $\lim u(z, \xi, \varsigma) \rightarrow+\infty$ as $|\operatorname{Re} \xi|^{2}+|\operatorname{Re} \varsigma|^{2} \rightarrow \infty$, for every $(z, \operatorname{Im} \xi, \operatorname{Im} \varsigma) \in U \times R^{2 q}$.

Assertion. If $u(z, w)$ is strictly $r$-plurisubharmonic, then $U(z, \xi, \zeta)$ is strictly $(r+q)$-plurisubharmonic.

To check this, consider $\left(z_{0}, \xi_{0}, \varsigma_{0}\right) \in U \times \mathbf{C}^{2 q}$ and $\left(z_{0}, w_{0}\right)=\left(z_{0}, T\left(\xi_{0}, \varsigma_{0}\right)\right)$. We have to show that Hess ${ }^{\mathbf{C}} U\left(z_{0}, \xi_{0}, \varsigma_{0}\right)$ has at most $(q+r)$ nonpositive eigenvalues. Clearly, it suffices to find a complex hyperplane $P_{r} \subset \mathbf{C}^{k+2 q}$ through $\left(z_{0}, \xi_{0}, \zeta_{0}\right)$, such that $\operatorname{dim}^{\mathbf{C}} P_{r}=k+q-r$ and $U \mid P_{r}$ is strictly plurisubharmonic near $\left(z_{0}, \xi_{0}, \varsigma_{0}\right)$.

Since $u(z, w)$ is strictly $r$-plurisubharmonic, there is a complex hyperplane $P \subset$ $\mathbf{C}^{k+q}$, through $\left(z_{0}, w_{0}\right)$, such that $\operatorname{dim} P=k+q-r$ and $u \mid P$ is strictly plurisubharmonic near $\left(z_{0}, w_{0}\right)$. Observe now that $T$ is $\mathbf{C}$-linear on the $q$-dimensional subspace $L$ of $\mathbf{C}^{2 q}$, where $L=\{(\xi, \varsigma): \xi=i \varsigma\}$. (One can check that $T(i \xi, i \varsigma)=i T(\xi, \zeta)$, if $(\xi, \varsigma) \in L$.) Now let $P_{r}=\left\{(z, \xi, \varsigma):(z, T(\xi, \varsigma)) \in P,(\xi, \varsigma) \in L+\left(\xi_{0}, \varsigma_{0}\right)\right\}$. The map $(z, \xi, \varsigma) \rightarrow(z, T(\xi, \varsigma)): P_{r} \rightarrow P$ is C-affine and bijective, and so $\operatorname{dim} P_{r}=\operatorname{dim} P=$ $k+q-r$ and $U \mid P_{r}$ is strictly plurisubharmonic at $\left(z_{0}, \xi_{0}, \varsigma_{0}\right)$. This completes the proof of the assertion.

Since $v(z)=\inf _{w} u(z, w)=\inf _{\xi, \varsigma} U(z, \xi, \varsigma)$ and $U(z, \xi, \varsigma)$ satisfies all the assumptions of Lemma 5.3, $v(z)$ is strictly $(q+r)$-plurisubharmonic, because $U(z, \xi, \varsigma)$ is strictly $(q+r)$-plurisubharmonic. For $r=0$, this proves part (a).

The general case of nonnecessarily smooth, plurisubharmonic function $u(z, w)$ can be reduced to case (a) as in Kiselman [5, p. 142]. First, it is enough to consider $u(z, w)$ bounded from below, $\operatorname{since} \max (u(z, w),-N) \searrow u(z, w)$ as $N \rightarrow \infty$, and if $v_{N}(z)=\inf _{w} \max (u(z, w),-N)$ is $q$-plurisubharmonic, then so is $v(z)$, for $v_{N}(z) \searrow$ $v(z)$; cf. [12, Proposition 1.2(vii)]. Assume now that $u(z, w)$ is bounded from below and let $v_{\delta}(z)=\inf _{w}\left(u(z, w)+\delta|w|^{2}\right), \delta>0$. Similarly as above, it suffices to show that $v_{\delta} \in P_{q}$, since $v_{\delta}(z) \searrow v(z)$.

Fix $\delta>0$; shrinking $U$, if necessary, we can assume that the minimum of the function $w \rightarrow u(z, w)+\delta|w|^{2}$ is located within some ball $B(0, R) \subset \mathbf{C}^{q}$, with $R$ independent on $z$. Now let $\varphi_{\varepsilon}(z, w)$ be a standard smooth positive convolution kernel supported by an $\varepsilon$-ball, $\varepsilon>0$. Let $u^{\varepsilon}(z, w)=\left(u(z, w)+\delta|w|^{2}\right) * \varphi_{\varepsilon}+$ $\varepsilon\left(|z|^{2}+|w|^{2}\right)$. Fix a compact set $K \subset U$. By a standard argument, we can choose a sequence $\varepsilon(n) \searrow 0$, such that $n^{-1}+u_{\varepsilon(n)}(z, w) \searrow u(z, w)+\delta|w|^{2}$ on $K \times \bar{B}(0, R+1)$. Let $v^{n}(z)=\inf _{w}\left(u^{\varepsilon(n)}(z, w)+1 / n\right)$. By the choice of $R, v^{n}(z)=$ $\inf _{|w| \leq R+1}\left(u^{\varepsilon(n)}(z, w)+1 / n\right)$, and so $v^{n}(z) \searrow v_{\delta}(z), z \in K$. Since functions $u^{\varepsilon}(z, w)$ satisfy all the assumptions of Theorem 5.1, part (a), it follows that $v^{n}(z)$ are strictly $q$-plurisubharmonic on $K$ and so, $v_{\delta} \in P_{q}(\operatorname{Int} K)$. Q.E.D.

\section{Duality between interpolation methods.}

THEOREM 6.1. Let $M, \Gamma, Q, X, D$ be as in Definition 3.3. Let $\left(\mathbf{C}^{n}, p_{z}(\cdot)\right)_{z \in X}$ be a $P_{Q}^{c o}$-interpolation family of normed spaces. Then, the dual normed spaces form a $P_{Q^{d}}^{\mathrm{co}}$-interpolation family. 
The theorem will be proven in two steps. First, we prove it in the special (and extreme) case when $Q^{d}=P_{0}$, using the minimum principle (Theorem 5.1) of the last section. Then, we apply [20, Theorem 4.9] to reduce the general problem to the special case. To handle the special case, we specify first some of the results of $\S \S 3,4$ to the situation when $Q=P_{k-1}$ or $P_{0}\left(\right.$ where $\left.k=\operatorname{dim}^{C}(M \backslash \Gamma)\right)$.

REMARK 6.2 (CF. [20, REMARK 4.8]) If $Q=P_{q}$, then $P_{Q}^{\text {co }}=P_{q}^{\text {co }}$, where $P_{q}^{c o}$ consists of $q$-plurisubharmonic functions $u(z, w)$, which are convex in $w$. Thus $P_{q}^{\text {co }}$-interpolation family is understood as $P_{Q}^{\text {co }}$-interpolation family with $Q=P_{q}$, etc.

PROPOSITION 6.3. If $D$ is a complex manifold of dimension $k$ and $p_{z}, z \in D$, are norms on $\mathrm{C}^{n}$, such that the function $(z, w) \rightarrow 1 / p_{z}(w)$ is $(n-1)$-plurisubharmonic on $D \times\left(\mathbf{C}^{n} \backslash\{0\}\right)$, then $\left(\mathbf{C}^{n}, p_{z}(\cdot)\right)_{z \in D}$ form a $P_{k-1}^{\text {co }}$-superinterpolation family of normed spaces.

(It will be shown elsewhere that the sufficient condition given in this corollary is also necessary.)

ProOF. By Corollary 4.3, it suffices to show that $u \in\left(P_{k-1}^{\text {co }}\right)^{d}\left(D \times\left(\mathbf{C}^{n} \backslash\{0\}\right)\right)$, where $u(z, w)=\min \left(-\log p_{z}(w), 0\right)$; cf. [18, Definition 1.11]. Let $\varphi(t)=\log t$, for $t \in(0,1]$, and $\varphi(t)=0$ for $t \geq 1$. Then $\varphi:(0, \infty) \rightarrow R$ is nondecreasing and $u(z, w)=\varphi\left(1 / p_{z}(w)\right), w \neq 0$. Since the function $(z, w) \rightarrow 1 / p_{z}(w)$ is $(n-1)$ plurisubharmonic, $u(z, w)$ is $n$-plurisubharmonic, by Assertion 2 in the proof of Lemma 2.8. By this and the obvious inclusion $P_{k-1}^{\text {co }} \subset P_{k-1}$, we get $u+P_{k-1}^{\text {co }} \subset P_{n}+$ $P_{k-1} \subset P_{n+k-1}$, by [12, Theorem 5.1]. Since the latter class has local maximum property (cf. [12, Proposition $1.2\left(\right.$ viii)]), $u \in\left(P_{k-1}^{c o}\right)^{d}$, as required. Q.E.D.

The following characterization follows immediately from Remark 6.2 and Theorems 3.8 and 4.1 .

PROPOSITION 6.4. Let $D$ be a complex manifold of dimension $k$. Then each of the following conditions is sufficient and necessary in order that normed spaces $\left\{\mathbf{C}^{n}, p_{z}(\cdot)\right\}_{z \in D}$ form a $P_{0}^{\text {co }}$-subinterpolation family:

(i) the function $(z, w) \rightarrow \log p_{z}(w)$ is plurisubharmonic on $D \times \mathbf{C}^{n}$; and

(ii) the set $\left\{(z, w) \in D \times \mathbf{C}^{n}: p_{z}(w)<1\right\}$ is locally pseudoconvex in $D \times \mathbf{C}^{n}$ (in the usual sense).

PROPOSITION 6.5. If $D$ is a complex manifold of dimension $k, 0 \leq q \leq k-1$, and $\left\{\mathbf{C}^{n}, p_{z}\right\}_{z \in D}$ is a $P_{q}^{\text {co }}$-superinterpolation family of normed spaces, then the dual spaces form a $P_{k-1-q}^{\text {co }}$-subinterpolation family.

ProOF. Apply Corollary 4.4 to $Q=P_{q}$ and note that $Q^{d}=P_{k-q-1}$ (cf. (1.17)) and $P_{Q}^{\text {co }}=P_{q}^{\text {co }}, P_{Q^{d}}^{\text {co }}=P_{k-q-1}^{\text {co }}($ cf. Remark 6.2). Q.E.D.

THEOREM 6.6. Let $D$ be a complex manifold of dimension $k$ and $\left\{\mathbf{C}^{n}, p_{z}\right\}_{z \in D}$ be a $P_{0}^{c o}$-subinterpolation family of normed spaces. Then the dual normed spaces form a $P_{k-1}^{\mathrm{co}}$-superinterpolation family.

PROOF. Let $q_{z}: \mathbf{C}^{n} \rightarrow R$ be the dual norms to $p_{z}(\cdot)$ and let $v(z, \xi)=1 / q_{z}(\xi)$, $z \in D, \xi \neq 0$. By Proposition 6.3, it suffices to show that $v \in P_{n-1}\left(D \times\left(\mathbf{C}^{n} \backslash\{0\}\right)\right)$.

Since $q_{z}(\xi)=\sup _{w \neq 0}\left(|\langle\xi, w\rangle| / p_{z}(w)\right)$ and since $p_{z}(w)$ is complex-homogeneous, we get $v(z, \xi)=\inf \left\{p_{z}(w):\langle w, \xi\rangle=1\right\}$. To show that $v(z, \xi)$ is $(n-1)$-plurisubharmonic near $(z, e), e \neq 0$, fix $e$ and choose a direct sum decomposition $\mathbf{C}^{n}=\mathbf{C} e+X_{0}$. 
Then let $W_{0}=\left\{w \in \mathbf{C}^{n}:\langle e, w\rangle=0\right\}$ and choose $f \in C^{n}$ such that $\langle e, f\rangle=1$ and $X_{0}=\left\{\xi \in \mathbf{C}^{n}:\langle\xi, f\rangle=0\right\}$. Observe now, that if $\xi=\alpha e+\xi_{0}, \xi_{0} \in X_{0}$, then the hyperplane $\{w:\langle\xi, w\rangle=1\}$ consists of all vectors $w=\alpha^{-1}\left(1-\left\langle\xi_{0}, w_{0}\right\rangle\right) e+w_{0}$, where $w_{0} \in W_{0}$. It is now clear that $v(z, \xi)=\inf \left\{\psi\left(z, \alpha, \xi_{0}, w_{0}\right): w_{0} \in W_{0}\right\}$, where $\xi=\alpha e+\xi_{0}$ and $\psi\left(z, \xi, w_{0}\right)=p_{z}\left(\alpha^{-1}\left(1-\left\langle\xi_{0}, w_{0}\right\rangle\right) e+w_{0}\right)$. By Proposition 6.4(i), $(z, w) \rightarrow p_{\boldsymbol{z}}(w)$ is a plurisubharmonic function, and so $\psi$ is plurisubharmonic as well. Clearly, for fixed $z, \alpha, \xi_{0}$, the function $\psi$ is convex in $w_{0}$. Since $\operatorname{dim} W_{0}=n-1$, Theorem 5.1 implies that the function $v(z, \xi)$ is $(n-1)$-plurisubharmonic for $\alpha \neq 0$, in particular near $(z, e)$. Q.E.D.

COROllary 6.7. If $D$ is a complex manifold of dimension $k$ and $\left\{\mathbf{C}^{n}, p_{z}\right\}_{z \in D}$ is a $P_{k-1}^{c o}$-subinterpolation family of normed spaces, then the dual normed spaces form a $P_{0}^{\mathrm{co}}$-superinterpolation family.

ProOf. Let $W(z)=\left\{w \in \mathbf{C}^{n}: p_{z}(w) \leq 1\right\}$. Clearly, $z \rightarrow W(z)^{0}$ is usc. To show that the polars $W(z)^{0}$ form a $P_{0}^{\text {co }}$-superinterpolation family, we check condition of Definition 3.3 with $G$ strictly pseudoconvex, $\bar{G} \subset D$.

Let $V: \bar{G} \rightarrow 2^{\mathbf{C}^{n}}$ be a $P_{0}^{\text {co }}$-interpolation family, such that $W(\varsigma)^{0} \subseteq V(\varsigma), \varsigma \in \partial G$. Since $\{V(z)\}_{z \in \bar{G}}$ is, in particular, a $P_{0}^{\text {co }}$-subinterpolation family, $\left\{V(z)^{0}\right\}_{z \in \bar{G}}$ is a $P_{k-1}^{\text {co }}$-superinterpolation family. If $K: \bar{G} \rightarrow 2^{\mathrm{C}^{n}}$ is the $P_{k-1}^{\text {co }}$-interpolation family with boundary data $V(\varsigma)^{0}$ (which exists by Theorem 2.3), then $V(\varsigma)^{0}=K(\varsigma) \subset$ $W(\varsigma)$, and so $V(z)^{0} \subset K(z)$ (by $P_{k-1}^{\text {co }}$-superinterpolation property of $V(z)$ ) and $K(z) \subset W(z)$ (by $P_{k-1}^{\text {co }}$-subinterpolation property of $\left.W(\cdot)\right)$. Thus, $V(z)^{0} \subset W(z)$, and so $W(z)^{0} \subset V(z), z \in \bar{G}$. Q.E.D.

The last two results imply immediately the following.

COROLlaRY 6.8. Theorem 6.1 is true when $Q=P_{0}$ or $Q=P_{k-1}$, where $k=\operatorname{dim}^{\mathbf{C}} M$. Specifically, if $\left\{\mathbf{C}^{n}, p_{z}\right\}_{z \in X}$ and $\left\{\mathbf{C}^{n}, q_{z}\right\}_{z \in X}$ are dual families of normed spaces, then one of them is a $P_{0}^{\mathrm{co}}$-interpolation family, if and only if the other is a $P_{k-1}^{c o}$-interpolation family.

THEOREM 6.9. Let $M, \Gamma, Q, X, D$ as in Definition 3.3. Let $z \rightarrow K(z): X \rightarrow$ $2^{\mathrm{C}^{n}}$ be a family of norming bodies. Then

(a) $K(\cdot)$ is a $P_{Q}^{\mathrm{co}}$-superinterpolation family on $X$ if and only if, for every function $g \in Q(H), H \subset X, H$ relatively open in $X$, the norming bodies $e^{g(z)} K(z)_{z \in H}$ form a $P_{0}^{\mathrm{co}}$-superinterpolation family on $H$; and

(b) $K(\cdot)$ is a $P_{Q}^{\text {co }}$-subinterpolation family on $X$, if and only if for every $g \in$ $Q^{d}(H), H \subset X, H$ relatively open in $X$, the norming bodies $\left\{e^{-g(z)} K(z)\right\}_{z \in H}$ form a $P_{k-1}^{c o}$-subinterpolation family.

PROOF. (a) (Sufficiency) If $p_{z}(w)$ denotes the Minkowski functional corresponding to $K(z)$, then $w \rightarrow e^{-g(z)} p_{z}(w)$ is the norm corresponding to the norming body $e^{g(z)} K(z)=\left\{e^{g(z)} w: w \in K(z)\right\}$. Since $z \rightarrow e^{g(z)} K(z)$ is a $P_{0}^{\text {co }}$-superinterpolation family when $g \in Q$, Corollary $4.3\left(\right.$ iii) implies that $u \in\left(P_{0}^{\text {co }}\right)^{d}\left(V \times\left(\mathbf{C}^{n} \backslash\{0\}\right)\right)$, where $u(z, w)=\varphi\left(-\log \left(e^{-g(z)} p_{z}(w)\right)\right)$, for every $\varphi \in C^{(2)}(R)$ with $\varphi^{\prime}(x)>0$ on $R$. Thus, for every such $\varphi$ and $g \in Q$, the function $u(z, w)=\varphi\left(g(z)-\log p_{z}(w)\right)$ is of class $\left(P_{0}^{\text {co }}\right)^{d}$. By [20, Theorem 4.9], these properties imply that the function $(z, w) \rightarrow \varphi\left(-\log p_{z}(w)\right)$ is of class $\left(P_{Q}^{c o}\right)^{d}$ and so, by Corollary $4.3(\mathrm{iii}), z \rightarrow K(z)$ is a $P_{Q}^{c o}$-superinterpolation family, as required. 
(a) (Necessity) If $h(z)$ is a pluriharmonic function on $M$, consider a (locally defined) holomorphic function $F(z)$, such that $\operatorname{Re} F(z)=h(z)$. By the invariance of the class $P_{Q}^{\text {co }}$ with respect to the map $(z, w) \rightarrow\left(z, e^{F(z)} w\right)$, cf. $(1.9)(\mathrm{v})$, the norming bodies $\left\{e^{h(z)} K(z)\right\}_{z \in X}$ form a $P_{Q}^{\text {co }}$-superinterpolation family, and so, by Corollary $4.3(\mathrm{iii}),(z, w) \rightarrow \varphi\left(h(z)-\log p_{z}(w)\right)$ is a function of class $\left(P_{Q}^{\text {co }}\right)^{d}$, for every $\varphi \in C^{(2)}(R)$ with $\varphi^{\prime}(x)>0, x \in R$. This being true for arbitrary pluriharmonic $h$, [20, Theorem 4.9(iii)] implies that for every $g \in Q$, the function $\varphi\left(g(z)-\log p_{z}(w)\right)$ is of class $\left(P_{0}^{\text {co }}\right)^{d}$. In view of the earlier remarks, Corollary 4.3 (iii) implies that $z \rightarrow e^{g(z)} K(z)$ is a $P_{0}^{\text {co }}$-superinterpolation family.

(b) (Necessity) If $p_{z}(w)$ is the Minkowski functional of $K(z)$, then $w \rightarrow e^{g(z)} p_{z}(w)$ is the Minkowski functional determined by the norming body $e^{-g(z)} K(z)$. If $K(\cdot)$ is a $P_{Q}^{\text {co }}$-subinterpolation family on $X$, then the function $(z, w) \rightarrow \log p_{z}(w)$ belongs to $P_{Q}^{\text {psh }}$, by Theorem 4.1. By this and (1.14), $\log \left(e^{g(z)} p(z)\right)=g(z)+\log p_{z}(w)$ is a function of $P_{k-1}^{\text {psh }}$ class (cf. Definition 1.1 and Remark 6.2) and, by Theorem 4.1, $z \rightarrow e^{-g(z)} K(z)$ is a $P_{k-1}^{c o}$-subinterpolation family.

(b) (Sufficiency) Conversely, if $z \rightarrow e^{-g(z)} K(z)$ is a $P_{k-1}^{\text {co }}$-subinterpolation family, the function $g(z)+\log p_{z}(w)$ is of $P_{k-1}^{\text {psh }}$ class. Hence, if we fix a holomorphic $\operatorname{map} F: H \rightarrow \mathbf{C}^{n}, H \subset D$, and let $v(z)=\log p_{z}(F(z))$, we get $(v+g) \in P_{k-1}(H)$, for every $g \in Q^{d}(H)$, and so $v \in Q^{d d}$; cf. [18, Definition 1.11]. By (1.15), $v \in Q$, for every $F$, and so $(z, w) \rightarrow \log p_{z}(w)$ is of $P_{Q}^{\text {psh }}$ class by Definition 1.1. Hence, $\{K(z)\}_{z \in X}$ is a $P_{Q}^{\text {co }}$-subinterpolation family. Q.E.D.

Using the last result, we will obtain now the general duality result from the special case (Corollary 6.8).

COROLlaRY 6.10. In the notation of Theorem 6.1 , if $\left\{\mathbf{C}^{n}, p_{z}(\cdot)\right\}_{z \in X}$ form a $P_{Q}^{\mathrm{co}}$-subinterpolation family of normed spaces, then the dual normed spaces form a $P_{Q^{d}}^{\mathrm{co}}$-superinterpolation family.

Proof. Let $W(z)=\left\{w \in \mathbf{C}^{n}: p_{z}(w) \leq 1\right\}$. The unit balls of the dual norms are $W(z)^{0}$. One can prove easily that $z \rightarrow W(z)^{0}: X \rightarrow 2^{\mathrm{C}^{n}}$ is usc, provided $z \rightarrow W(z): X \rightarrow 2^{\mathrm{C}^{n}}$ is lsc. Now let $g \in Q^{d}(H), H \subset X, H$ relatively open in $X$. By Theorem $6.9(\mathrm{~b})$, the norming bodies $\left\{e^{-g(z)} W(z)\right\}_{z \in H}$ form a $P_{k-1^{-}}^{\text {co }}$ subinterpolation family and, by Corollary 6.7, their polars $\left\{e^{g(z)} W(z)^{0}\right\}_{z \in H}$ form a $P_{0}^{\text {co }}$-superinterpolation family. Since $g \in Q^{d}$ is arbitrary, Theorem $6.9(\mathrm{a})$ implies that $z \rightarrow W(z)^{0}$ is a $P_{Q^{d}}^{\text {co }}$-superinterpolation family on $X$. Q.E.D.

Proof OF THEOREM 6.1. Since a family of normed spaces $\left\{\mathbf{C}^{n}, p_{z}\right\}$ is a $P_{Q^{d^{-}}}^{\text {co }}$ interpolation family, if and only if it is both a super- and subinterpolation family of class $P_{Q^{d}}^{\text {co }}$, Corollaries 4.4 and 6.10 imply immediately Theorem 6.1. Q.E.D.

\section{Examples. Biholomorphically invariant interpolation methods.}

7.1. Comparison of interpolation methods. (a) For every class $Q$ under consideration, $P_{0} \subset Q \subset P_{k-1}$. If $G$ is strictly pseudoconvex, then a given continuous family $\{B(\varsigma)\}_{\varsigma \in \partial G}$ of norming bodies can be extended to a $P_{Q}^{c o}$-interpolation family, say $\left\{W_{Q}(z)\right\}_{z \in \bar{G}}$. If $Q=P_{q}$, denote this interpolation by $W_{q}(z), z \in \bar{G}$. The following inclusions hold: $W_{k-1}(z) \subset W_{Q}(z) \subset W_{0}(z) ; W_{k-1}(z) \subset W_{k-2}(z) \subset \cdots \subset W_{1}(z) \subset$ $W_{0}(z), z \in \bar{G}$. 
(b) In one complex dimension all these methods are identical and, in case $G=$ the unit disc, produce the same result as the procedure of Coifman et al. $[\mathbf{1}, \mathbf{2}]$. This follows from [14, Theorem 4].

(c) Proof of Corollary 6.8, when specialized to the planar case, gives a new proof of the duality theorem in Coifman et al. [1].

In addition, Theorem 6.6 answers the problem posed, for $k=1$, by Rochberg [ 8 , Conjecture, p. 365].

(d) All the methods $P_{Q}^{\text {co }}$ are invariant with respect to biholomorphic bundle automorphisms $(z, w) \rightarrow(z, T(z) w)$, with $T(z)$ affine. In contrast, the $P_{q}^{\text {co }}$-interpolation methods, $q=0,1, \ldots, k-1$, are also invariant with respect to biholomorphic maps on the base space, i.e. $(z, w) \rightarrow(f(z), w)$. They are characterized by this property (among $P_{Q}^{\mathrm{co}}$ methods).

7.2. $P_{q}^{\mathrm{co}}$-interpolation methods. (a) Existence. Let $0 \leq q \leq k-1, k=$ $\operatorname{dim}_{\mathbf{C}}(M \backslash \Gamma)$, and let $r=\min (q, k-q-1)$. Assume that $G \subset M \backslash \Gamma$ is $P_{r}$-regular and $\left\{\mathbf{C}^{n}, p_{\zeta}\right\}$ is a continuous family of normed spaces on $\partial G$. By Theorem 2.3, continuous $P_{s}^{c o}$-interpolation of these boundary data exists for $r \leq s \leq k-r-1$.

(b) Duality. By Theorem 6.1, the $P_{q}^{\text {co }}$-interpolation of given spaces $\left\{\mathbf{C}^{n}, p_{\varsigma}\right\}_{\varsigma} \in \partial G$ and $P_{k-q-1}^{c o}$-interpolation of spaces dual to $\left\{\mathbf{C}^{n}, p_{\varsigma}\right\}_{\varsigma \in \partial G}$ form mutually dual families of normed spaces.

In particular, for $k$ odd, the $P_{m}^{\text {co }}$-interpolation method, where $m=\frac{1}{2}(k-1)$, is selfdual.

\section{Examples. Subharmonic interpolation.}

8.1. Axiomatic subharmonicity. The main duality result (Theorem 6.1) is particularly plausible in case $Q=Q^{d}$, for then the $P_{Q}^{\text {co }}$-interpolation method turns out to be self-dual. The result on interpolation of operator norm (Corollary 4.5) yields most satisfactory conclusions in case $(A Q)^{d}=Q$, i.e. $Q^{d}$ is closed with respect to addition. In the context of this paper, these two conditions are equivalent to $Q^{d}=Q, Q+Q=Q$ and imply that $Q=$ the class of all subharmonic functions on $M$ (in an appropriate sense).

8.2. The Euclidean case: $M=\mathbf{C}^{k}$. As it follows from [19], the only translationinvariant pseudoconvex classes $Q$ on $\mathbf{C}^{k}$ satisfying (1.0)-(1.12) are classes of all subharmonic functions relative to a given Hermitian product on $\mathbf{C}^{k}$, the usual subharmonic class being the principal example.

(a) Existence. By Theorem 2.3, if $G \subset \mathbf{C}^{k}$ is an open, relatively compact set, regular in the usual sense of potential theory on $\mathrm{C}^{k}=R^{2 k}$, then every prescribed continuous family $\left\{\mathbf{C}^{n}, p_{\varsigma}\right\}, \varsigma \in \partial G$, of normed spaces, extends uniquely to a continuous, $P_{\text {subh }}^{\text {co }}$-interpolation family $\left\{\mathbf{C}^{n}, \tilde{p}_{z}\right\}$ on $\bar{G}$.

(b) Selfduality of the subharmonic interpolation. By Theorem 6.1, the dual normed spaces to $\left\{\mathbf{C}^{n}, \tilde{p}_{z}\right\}$ form a $P_{\text {subh }}^{\text {co }}$-interpolation family extending the dual spaces to $\left\{\mathbf{C}^{n}, p_{\varsigma}\right\}, \varsigma \in \partial G$.

8.3. Moebius-invariant subharmonic interpolation. The simplest non-Euclidean complex homogeneous space is the unit ball $B$ in $\mathbf{C}^{k}$. In our notation $M=\bar{B}$, $\Gamma=\partial B, M \backslash \Gamma=B=K / H$, where $K$ is the Moebius group of biholomorphic automorphisms of $B$ and $H$ is the unitary group. The unique class $Q$ on $M$, satisfying conditions (1.0)-(1.12) (in particular Moebius-invariant) and such that $Q^{d}=Q$ and $Q+Q \subset Q$, is the class of functions, usc at the points of $\partial B$ and 
$M$-subharmonic in $B$, in the sense of Rudin [11, Definition 3.3.6]. The meaning of the class $P_{M \text {-subh }}^{\mathrm{co}}$ is now clear.

Similarly as in 8.2(a), Theorem 2.3 gives existence of $P_{M \text {-subh }}^{\mathrm{co}}$-interpolation family for continuous data on $\partial G$, where $G$ is any relatively open subset of $\bar{B}$ which is regular with respect to Moebius-harmonic Dirichlet problem at the points of $\partial G \backslash \partial B$.

Self-duality of $M$-subharmonic interpolation holds in the same way as in 8.2(b).

8.4. Invariant subharmonic interpolation on other homogeneous spaces. In the general case $M \backslash \Gamma=K / H$, where $K$ and $H$ are real Lie groups, with $H$ compact. Then $Q$ is the class of all subharmonic functions with respect to an invariant Laplacian. Below, we denote any such class by "subh". Properties 8.2(a) and (b) extend in obvious ways to $P_{\text {subh }}^{\mathrm{co}}$-interpolation on complex homogeneous spaces (cf. Theorems 2.3 and 6.1).

8.5. Operator norm in case of subharmonic interpolation. By Corollary 4.5, if $\left\{\mathbf{C}^{n}, p_{z}\right\}$ and $\left\{\mathbf{C}^{m}, q_{z}\right\}$ are $P_{\mathrm{subh}^{\mathrm{co}}}$-interpolation families on $G \subset M \backslash \Gamma=K / H$ and $z \rightarrow T(z): G \rightarrow L\left(\mathbf{C}^{n}, \mathbf{C}^{m}\right)$ is an analytic family of operators, then $z \rightarrow \log \left\|T_{z}\right\|$ is a subharmonic function on $G$.

8.6. Characterization of subharmonic interpolation. If $p_{z}^{*}$ are the dual norms to $p_{z}, z \in D$, then $\left\{\mathbf{C}^{n}, p_{z}\right\}_{z \in D}$ form a $P_{\text {subh }}^{\text {co }}$-interpolation family, if and only if for arbitrary analytic mappings $F_{i}: H_{i} \rightarrow \mathbf{C}^{n}, H_{i} \subset D, i=1,2$, the functions $z \rightarrow p_{z}\left(F_{1}(z)\right)$ and $z \rightarrow p_{z}^{*}\left(F_{2}(z)\right)$ are subharmoinic. (Follows from Theorem 4.1 and 6.1.)

Concluding remarks. There are many extensions and applications of the results of this paper. In particular, interpolation families can be also characterized in terms of curvature of the norm function $p_{z}(w)$ (cf. $[\mathbf{2 0}, \S 4]$ ) in the spirit of Kobayashi [6] and Rochberg [8]. Theorem 6.6 gives a partial positive answer to a problem of Kobayashi $[\mathbf{6}, \S 5]$. In case of subharmonic interpolation, a simpler proof of duality can be given. The details will be described in a subsequent paper.

This paper has some history. In the initial version, completed in Winter and Spring 1985, the analogous results were obtained for $P_{q}^{\text {co }}$-interpolation methods. They were discussed during the author's visit in St. Louis in March 1985 and presented at the Banach space Conference, Kent, August 1985, and, in more detail, in Indianapolis, April 1986 (cf. [16]), but their publication was delayed for accidental reasons (the manuscript was lost by an airline). In Winter and Spring 1986 they were generalized to the present form, in which they were reported in Toledo, July 1986, (cf. [17]).

Recently, the author has received the reports of Rochberg [25], and Coifman and Semmes [24]. Their work is related to this one in the part concerning subharmonic interpolation in $\mathbf{C}^{k}$. In this case, they obtained (by different methods) results on the existence, uniqueness, continuity and reiteration of interpolation families, and on interpolation of operators, which follow from more abstract conclusions of this paper (Theorem 2.3, Remark 2.10 and Corollaries 4.5, 4.7). There are many other interesting problems, which are covered in $[\mathbf{2 4}, \mathbf{2 5}]$, including interpolation of Hilbert spaces and relations with P.D.E.'s, in particular Yang-Mills equation. On the other hand, our paper contains also duality theorems and group-invariant subharmonic interpolation on complex homogeneous spaces (which are listed as 
open problems in [25]), as well as biholomorphic invariant interpolation methods and interpolation of quasi-normed spaces.

ACKNOWledgments. The author is grateful to Professors R. Coifman, R. Rochberg and G. Weiss for their interest in this work. In fact, it was Richard Rochberg and Guido Weiss who attracted his attention to the interpolation problem in several dimensions.

ADDED IN PROOF. Further results related to $\S 8$ are obtained in the following papers of this author: Complex interpolation of normed and quasinormed spaces in several dimensions. Part II. Properties of harmonic interpolation, and Part III. Regularity results for harmonic interpolation.

\section{REFERENCES}

1. R. Coifman, M. Cwikel, R. Rochberg, Y. Sagher and G. Weiss, The complex method for interpolation of operators acting on families of Banach spaces, Lecture Notes in Math., vol. 779, Springer-Verlag, Berlin and New York, 1980, pp. 123-153.

2. _ A theory of complex interpolation for families of Banach spaces, Adv. in Math. 33 (1982), 203-229.

3. R. L. Hunt and J. J. Murray, q-plurisubharmonic functions and a generalized Dirichlet problem, Michigan Math. J. 25 (1978), 299-316.

4. C. O. Kiselman, The partial Legendre transformation for plurisubharmonic functions, Invent. Math. 49 (1978), 137-148.

5. _ How smooth is the shadow of a smooth convex body? preprint.

6. S. Kobayashi, Negative vector bundles and complex Finsler structures, Nagoya Math. J. 57 (1975), 153-166.

7. R. Narasimhan, Analysis on real and complex manifolds, North-Holland, Amsterdam, 1968.

8. R. Rochberg, Interpolation of Banach spaces and negatively curved vector bundles, Pacific J. Math. 110 (1984), 355-376.

9. $\ldots$, Function theoretic results for complex interpolation families of Banach spaces, Trans. Amer. Math. Soc. 284 (1984), 745-758.

10. R. Rochberg and G. Weiss, Some topics in complex interpolation theory, Topics in Modern Harmonic Analysis, Ist. Naz. Alta Mat. F. Severi, Roma, 1983, pp. 769-818.

11. W. Rudin, Function theory in the unit ball of $C^{n}$, Springer-Verlag, New York, 1980.

12. Z. Slodkowski, The Bremermann-Dirichlet problem for q-plurisubharmonic functions, Ann. Scuola Norm. Sup. Pisa, Cl.-Sci. (4) 11 (1984), 303-326.

13. __ Local maximum property and q-plurisubharmonic functions in uniform algebras, J. Math. Anal. Appl. 115 (1986), 105-130.

14. _ Polynomial hulls with convex sections and interpolating spaces, Proc. Amer. Math. Soc. 96 (1986), 255-260.

15. __ An analytic set-valued selection and its applications to the corona theorem, to polynomial hulls and joint spectra, Trans. Amer. Math. Soc. 294 (1986), 367-377.

16. __ Complex interpolation families of normed spaces over several-dimensional parameter space. Abstracts of the Special Session in Several Complex Variables, 826th Meeting of the AMS, Indianapolis, April 1986.

17. __ On complex interpolation methods for families of normed spaces over domains in $\mathbf{C}^{k}$, Talk at International Conference on Harmonic Measure, Toldeo, Ohio, July, 1986.

18. __ Pseudoconvex classes of functions I. Pseudoconcave and pseudoconvex sets, Pacific J. Math. (to appear).

19. __ Pseudoconvex class of functions. II. Affine pseudoconvex classes on $R^{N}$ (submitted).

20. __ Pseudoconvex classes of functions. III. Characaterizations of dual pseudoconvex classes on complex homogeneous spaces. Trans. Amer. Math. Soc. (to appear).

21. _ Pseudoconvex classes of functions. IV (in preparation).

22. J. B. Walsh, Continuity of envelopes of plurisubharmonic functions, J. Math. Mech. 18 (1986), 143-148. 
23. T. Wolff, A note on interpolation spaces, Harmonic Analysis, Lecture Notes in Math., vol. 908, Springer-Verlag, Berlin and New York, 1982, pp. 199-204.

24. R. Coifman and S. Semmes, Interpolation of Banach spaces and non-linear Dirichlet problems, preprint.

25. R. Rochberg, The work of Coifman and Semmes on complex interpolation, several complex variables and PDE's, US-Swedish Seminar on Function Spaces and Applications, Lund, June, 1986 (to appear).

Department of Mathematics, University of Illinois at Chicago, Chicago, ILLINOIS 60680 